\title{
Midbrain Dopaminergic Neuron Development at the Single Cell Level: In vivo and in Stem Cells
}

\section{Emilía Sif Ásgrímsdóttir and Ernest Arenas*}

Division of Molecular Neurobiology, Department of Medical Biochemistry and Biophysics, Karolinska Institutet, Stockholm, Sweden

Parkinson's disease $(\mathrm{PD})$ is a progressive neurodegenerative disorder that predominantly affects dopaminergic (DA) neurons of the substantia nigra. Current treatment options for PD are symptomatic and typically involve the replacement of DA neurotransmission by DA drugs, which relieve the patients of some of their motor symptoms. However, by the time of diagnosis, patients have already lost about $70 \%$ of their substantia nigra DA neurons and these drugs offer only temporary relief. Therefore, cell replacement therapy has garnered much interest as a potential treatment option for PD. Early studies using human fetal tissue for transplantation in PD patients provided proof of principle for cell replacement therapy, but they also highlighted the ethical and practical

OPEN ACCESS

Edited by:

Emmanouil Metzakopian, University of Cambridge,

United Kingdom

Reviewed by:

Nilima Prakash,

Hamm-Lippstadt University of Applied

Sciences, Germany

Dan Lindholm,

University of Helsinki, Finland

*Correspondence: Ernest Arenas

ernest.arenas@ki.se

Specialty section:

This article was submitted to

Stem Cell Research,

a section of the journal

Frontiers in Cell and Developmental

Biology

Received: 02 March 2020

Accepted: 19 May 2020

Published: 25 June 2020

Citation:

Ásgrímsdóttir ES and Arenas E (2020) Midbrain Dopaminergic Neuron Development at the Single Cell Level: In vivo and in Stem Cells.

Front. Cell Dev. Biol. 8:463.

doi: 10.3389/fcell.2020.00463 difficulties associated with using human fetal tissue as a cell source. In recent years, advancements in stem cell research have made human pluripotent stem cells (hPSCs) an attractive source of material for cell replacement therapy. Studies on how DA neurons are specified and differentiated in the developing mouse midbrain have allowed us to recapitulate many of the positional and temporal cues needed to generate DA neurons in vitro. However, little is known about the developmental programs that govern human DA neuron development. With the advent of single-cell RNA sequencing (scRNA-seq) and bioinformatics, it has become possible to analyze precious human samples with unprecedented detail and extract valuable high-quality information from large data sets. This technology has allowed the systematic classification of cell types present in the human developing midbrain along with their gene expression patterns. By studying human development in such an unbiased manner, we can begin to elucidate human DA neuron development and determine how much it differs from our knowledge of the rodent brain. Importantly, this molecular description of the function of human cells has become and will increasingly be a reference to define, evaluate, and engineer cell types for PD cell replacement therapy and disease modeling.

Keywords: dopaminergic neuron, stem cell, progenitor, radial glia, single cell, machine learning, cell replacement, Parkinson's disease

\section{INTRODUCTION}

Parkinson's disease (PD) is the second most common neurodegenerative disorder, and its prevalence continues to increase as the population ages (Poewe et al., 2017). Clinically, PD is characterized by a classical triad of motor symptoms: rigidity, tremor, and bradykinesia (Lees et al., 2009). However, patients can also experience a broad spectrum of non-motor symptoms, 
including cognitive and sensory symptoms (Schapira et al., 2017). The two main pathological features of PD are the progressive loss of midbrain dopaminergic (mDA) neurons in the substantia nigra pars compacta $(\mathrm{SNc})$, and the intraneuronal accumulation of $\alpha$-synuclein enriched protein aggregates, termed Lewy bodies and Lewy neurites. The loss of mDA neurons in the SNc primarily affects the nigrostriatal pathway, resulting in a nigrostriatal dopamine deficiency, which causes many of the motor symptoms associated with PD (Fahn, 2003).

Current treatment options for PD aim to correct the loss of striatal dopamine through pharmaceutical intervention, either with drugs that modulate DA neurotransmission or that increase dopamine levels in the brain, such as L-DOPA. As an alternative treatment, patients that develop drug-related complications can have electrodes surgically implanted for deep brain stimulation, which compensate for the loss of DA neurotransmission by inhibiting excitatory neurotransmission. However, all of these treatments are symptomatic, and they neither address the underlying cause of the disease nor prevent the progressive degeneration of $\mathrm{mDA}$ neurons. Moreover, as the disease progresses, these treatments lose efficacy and lead to undesirable side-effects, further highlighting the need for PD treatment strategies that address the underlying cause of the disease, rather than its symptoms (Brichta et al., 2013).

In addition to novel therapies that address the underlying cause of $\mathrm{PD}$, there is a clear need for regenerative treatments capable of replacing the $\mathrm{mDA}$ neurons already lost in $\mathrm{PD}$ patients at the time of diagnosis. Therefore, cell replacement therapy is considered a promising treatment option as it could both provide a more physiological delivery of dopamine and replace other important functions of $\mathrm{mDA}$ neurons, such as the delivery of trophic factors, which can support other cell types within the basal ganglia that connect with $\mathrm{mDA}$ neurons. Human fetal ventral midbrain (VM) tissue has been successfully used for cell transplantation in clinical trials and has provided proof of concept for cell replacement therapy in PD (Arenas, 2010; Lindvall and Björklund, 2011). Notably, the patients who showed the most significant improvement after transplantation were able to discontinue their $\mathrm{PD}$ medication and have remained asymptomatic for over 20 years (Kefalopoulou et al., 2014). However, human fetal tissue is difficult to obtain, and differences in tissue quality, how the cells are prepared for transplantation, the surgical procedures employed, and differences in immunosuppression strategies after transplantation have led to variable results. These include a lack of improvement, and, in some cases, undesirable side effects such as graft-induced dyskinesias (Barker et al., 2015). These studies have led to a new clinical trial using human fetal VM tissue (TRANSEURO, NCT01898390), which has been designed to capture all the variables underlying the success of previous clinical trials. TRANSEURO is expected to facilitate the identification of optimal variables for cell transplantation and pave the way for the development of more efficient cell transplantation protocols that may also be applicable to other cell preparations. Indeed, the widespread clinical use of human fetal tissue for transplantation in PD patients is severely limited by several factors including ethical concerns regarding the use of tissue from aborted human embryos, difficulties in standardizing cell composition and quality, as well as the limited availability of this type of tissue (Barker et al., 2015). Due to this, alternative donor sources of mDA neurons for PD cell replacement therapy, such as human pluripotent stem cells, have been explored and developed (Allan et al., 2010; Arenas et al., 2015a; Barker et al., 2015, 2019; Lindvall, 2016; Björklund and Lindvall, 2017; Studer, 2017; Sonntag et al., 2018).

Human pluripotent stem cells (hPSCs) have emerged as an ideal source of material for cell transplantation since they can expand indefinitely and provided the right cues, they can differentiate into any cell type of the body. However, to guide the differentiation of hPSCs into a specific cell type, it is necessary to have a detailed understanding of the developmental steps leading to the generation of that cell type. In the case of PD, mDA neurons are the cell type of interest, and their development has been intensively studied in recent years (Arenas et al., 2015a; Blaess and Ang, 2015; Bodea and Blaess, 2015; Brignani and Pasterkamp, 2017; Smidt, 2017; Brodski et al., 2019; Poulin et al., 2020). However, most of our current knowledge comes from studying mouse mDA neuron development and our knowledge of human midbrain development still remains quite limited.

In mice, $\mathrm{mDA}$ neurons arise from the VM region, after regional specification of the neural tube. This regionalization process is accomplished through the combined action of two important signaling centers: the floor plate and the isthmic organizer. In this review, we will briefly discuss these two signaling centers, but for a detailed description, the reader is directed to the many excellent previous reviews in this area, including (Joyner et al., 2000; Martínez, 2001; Wurst et al., 2001; Placzek and Briscoe, 2005; Wilson and Maden, 2005; Nakamura et al., 2008; Cohen et al., 2013).

The floor plate is a specialized glial structure located along the ventral midline of the developing neural tube. This area has classically been considered a structural, non-neurogenic region that acts as a signaling center and contains radial glia cells, which secrete morphogens, such as Sonic hedgehog (Shh), that pattern the neural tube in the dorsoventral axis and specify neural identities (Placzek and Briscoe, 2005). However, fate-mapping studies have shown that unlike other floor plate radial glia, radial glia cells in the midbrain floor plate can undergo neurogenesis to give rise to mDA neurons (Bonilla et al., 2008). The second signaling center, the isthmic organizer (IsO), is located at the midbrain-hindbrain boundary and is established through the coordinated expression and mutual repression of the transcription factor Otx2 in the midbrain (Millet et al., 1996; Broccoli et al., 1999) and Gbx2 in the hindbrain (Wassarman et al., 1997; Millet et al., 1999). The IsO secretes the morphogens Wnt1 on the midbrain side and Fgf8 on the hindbrain side (Joyner et al., 2000; Puelles et al., 2004), which induces the expression of Wnt 1 in the VM floor plate; a necessary step for the establishment of the midbrain progenitor domain and for $\mathrm{mDA}$ neurogenesis (Joyner et al., 2000; Prakash et al., 2006; Andersson et al., 2013).

After specification, $\mathrm{mDA}$ progenitors residing in the ventricular zone (VZ) of the floor plate begin to express two transcription factors required for $\mathrm{mDA}$ neuron development, 
Foxa2 (Ferri et al., 2007) and Lmx1a (Andersson et al., 2006b). These progenitors then expand and subsequently undergo neurogenesis, a process regulated by Neurog2 (Kele et al., 2006) that results in the generation of post-mitotic $\mathrm{mDA}$ neuroblasts expressing the transcription factor Nr4a2 (Nurr1) (Zetterström et al., 1997). Following neurogenesis, mDA neuroblasts migrate, first in a radial manner following the processes of the radial glia and subsequently in a tangential manner, toward their final destinations in the mantle zone (MZ) (Kawano et al., 1995). During this migration process, the neuroblasts continue to differentiate and acquire the expression of transcription factors required for $\mathrm{mDA}$ neuron development such as $P b \times 1$ (Villaescusa et al., 2016) and Pitx3 (Smidt et al., 2004; Maxwell et al., 2005; Veenvliet et al., 2013), as well as genes that identify $\mathrm{mDA}$ neurons and are necessary for their function, including the rate-limiting enzyme for dopamine synthesis, tyrosine hydroxylase (Th) (Molinoff and Axelrod, 1971), the vesicular monoamine transporter (Vmat2/Slc18a2), and the dopamine transporter (Dat/Slc6a3) (Miller et al., 1999).

While human mDA neuron development is thought to follow similar principles, little is currently known about the cell type composition of the human VM and the molecular programs that govern human mDA neuron development. Histological analysis of human fetal midbrain tissue has provided some insights into the spatial and temporal organization of the human $\mathrm{VM}$ and $\mathrm{mDA}$ neuron development. The spatial organization of the human VM appears to correspond principally with the organization of the murine $\mathrm{VM}$, with the three layers of the ventricular, intermediate and mantle zones (VZ, IZ, and MZ) clearly identifiable. Furthermore, human mDA neuron development also appears to follow a similar sequence of events to that of murine mDA neuron development. For instance, the human midbrain floor plate is defined by the expression of LMX1A and FOXA2, with the FOXA2 domain extending further laterally into the basal plate (Nelander et al., 2009; Marklund et al., 2014). Moreover, in the VZ of the human floor plate, the pro-neural factor NEUROG2 overlaps with the expression of $L M X 1 A$, and in the IZ and MZ, cells expressing key determinants of post-mitotic mDA neurons, NR4A2, PITX3, and TH, can be observed (Nelander et al., 2009).

The information gained from studying $\mathrm{mDA}$ neuron development, whether that be in the murine or human VM, has in recent years led to the development of several protocols that direct the differentiation of hPSCs into functional mDA neurons capable of rescuing motor deficits in rodent and non-human primate models of PD (Kriks et al., 2011; Kikuchi et al., 2017; Kirkeby et al., 2017), as well as protocols compliant with good manufacturing practice (GMP) (Doi et al., 2014; Kirkeby et al., 2017; Nolbrant et al., 2017). Last year, following successful preclinical trials in animal models of $\mathrm{PD}$, the first clinical trial using human induced pluripotent stem cells (hiPSCs) as a cell source for transplantation was initiated (Takahashi and Price-Evans, 2019) and other trials using human embryonic stem cells are impending (Barker et al., 2017; Studer, 2017; Sonntag et al., 2018). However, though hPSC-derived mDA neurons have already entered clinical trials, the composition and quality of the cell preparations used or aimed for transplantation have not been fully elucidated at the single-cell level.

With the advent of single-cell RNA sequencing (scRNA-seq), it has become possible to profile the transcriptome of every individual cell in a tissue and to define their molecular signatures in an unbiased and systematic manner. Moreover, analyzing cells at different stages of development by scRNA-seq can provide a description of developmental processes at an unprecedented depth and resolution (Linnarsson and Teichmann, 2016). Recent studies have examined the cellular diversity of $\mathrm{mDA}$ neurons at the single cell level in mouse or in human. Microarray or scRNA-seq analysis of postnatal murine mDA neurons (Poulin et al., 2014; La Manno et al., 2016; Hook et al., 2018; Kramer et al., 2018; Saunders et al., 2018; Tiklová et al., 2019a) has led to the identification of 5-7 murine mDA neuron cell types and novel marker genes (reviewed in Poulin et al., 2020). However, the molecular diversity of the adult human brain remains largely to be explored and so far only the two previously known human adult $\mathrm{mDA}$ cell types, with substantia nigra $(\mathrm{SNc})$ and ventral tegmental area (VTA) phenotypes, have been described (Nichterwitz et al., 2016).

Less is known about the development of embryonic mDA neurons at the single cell level. While Kee et al. (2017) and Hook et al. (2018) detected embryonic mDA neurons in the murine midbrain, no subtypes of embryonic $\mathrm{mDA}$ neurons were identified. In contrast, our analysis of murine and human midbrain development unraveled the presence of three embryonic mDA neuron subtypes in both species (La Manno et al., 2016). Moreover, our study provided a first classification of the cell types in the developing murine and human VM, identifying both novel cell types and marker genes; thus, providing new insights into early mDA neuron development and the diversification of the $\mathrm{mDA}$ lineage into different embryonic mDA neuron subtypes. Additionally, in the study by La Manno et al. (2016), a systematic comparison of scRNA-seq data of human and murine development was performed, allowing for the comparison of the human and murine VM at the single-cell level. This study provided the first unbiased and systematic classification of the cell types in the developing human midbrain and made it possible to identify differences between human and murine midbrain development.

In the next sections, we focus on the differences between human and murine midbrain development as identified by scRNA-seq. These include differences in cell-type composition, temporal dynamics of development, and the expression of transcription factors at the single-cell level. Furthermore, we describe how the knowledge gained from scRNA-seq analysis can be used to assess the quality of DA neurons generated in vitro from hPSCs as well as to guide the improvement of $\mathrm{mDA}$ neuron differentiation and reprogramming protocols. We argue that a detailed single-cell level knowledge of the cell preparations being used for cell replacement therapy is necessary to identify the cell types required for functional replacement as well as any unnecessary or undesirable cell types in the preparation. We expect that such knowledge will improve the therapeutic potential and safety of future cell preparations for cell replacement in PD and that the strategy followed here will be 
useful in addressing the challenge of performing cell replacement in other tissues or organs.

\section{HUMAN MDA NEURON DEVELOPMENT AT THE SINGLE CELL LEVEL}

We recently used scRNA-seq to analyze and compare the human and murine $\mathrm{VM}$ at different stages of development, covering $\mathrm{mDA}$ neuron specification, neurogenesis and differentiation in both the human (weeks 6-11) and the mouse (E11.5 - E18.5) (La Manno et al., 2016). We used the Fluidigm C1 system, STRT-Seq and Back-SPIN to analyze a total of 1977 VM cells from 10 human embryos and 1907 cells from 271 murine embryos. From this analysis, 25 cell types were identified in the human VM, whereas 26 cell types were identified in the murine VM. The cell types identified in the human and murine VM included early proliferating cells such as radial glia and progenitors; post-mitotic cells, such as neuroblasts and neurons; as well as microglia and cells comprising the vasculature. In the following sections, we describe in greater detail some of the cell types identified in the developing human VM and discuss how these cell types compare to their homologous cell types in the murine VM.

However, it should be noted that while our scRNA-seq analysis provided the first unbiased classification of cell types in the developing human VM, it remains to be determined whether sampling a greater number of cells from additional human embryos will lead to the identification of additional cell types or a better definition of the cell types described in this review. As such, efforts are currently underway to sequence a greater number of cells and embryos, at additional developmental stages, using new and improved scRNA-seq methods, which are expected to improve the resolution of the analysis described here.

\section{Human VM Radial Glia}

Our analysis of the developing human VM (weeks 6-11) identified a greater diversity of radial glia cell types compared to the mouse (embryonic days 11.5-18.5) (La Manno et al., 2016) and to what has previously been described (Ostrem et al., 2017). While three distinct types of radial glia cells (Rgl1-3) were detected in the mouse VM, five molecularly defined radial glia cell types (Rgl1, 2a-c, 3) were found in the human VM by scRNA-seq. However, single-molecule fluorescent in situ hybridization (smFISH) analysis also revealed the presence of Rgl2c in the embryonic mouse VM, suggesting that other homologous human Rgl2 subtypes may also be present in the mouse VM. Further experiments will be necessary to determine whether equivalent midbrain $\mathrm{Rgl}$ cell types exist in both species and whether their spatial organization is similar.

Homologous radial glia cell types in both species were found to share the expression of certain genes, such as the neural stem/progenitor transcription factor (TF) Sox2 (Graham et al., 2003), and the expression of a fatty acid binding protein induced by Notch signaling (Fabp7) (Anthony et al., 2005) (Figure 1A). However, each radial glia cell type was found to have a distinctive expression profile, suggesting that they may serve separate functions during VM development. For instance, Rgl1, a cell type present in both the floor plate and the basal plate of the VZ during mouse VM development, is characterized by the expression of TFs implicated in neurogenesis and $\mathrm{mDA}$ neuron specification. Human and mouse Rgl1 share the expression of the pro-neural basic helix-loop-helix (bHLH) factor Ascl1, as well as the TF Otx2, both of which have many roles in $\mathrm{mDA}$ neuron development, from specification to neurogenesis (Figure 1A) (Vernay et al., 2005; Kele et al., 2006; Prakash et al., 2006; Omodei et al., 2008). Additionally, human Rgl1 express other essential determinants of mDA neurons such as the TFs LMX1A and $M S X 1$, which are required for the specification and differentiation of mDA neurons (Andersson et al., 2006b). Surprisingly, these TFs are not expressed in mouse Rgl1, but rather in a neuronal progenitor cell type present in the mouse VM (see below), suggesting that non-homologous cell types may serve the same function. Thus, while homologous Rgll may play a common role in specification and neurogenesis, their role in $\mathrm{mDA}$ neuron development may differ.

The second radial glia cell type, Rgl2, is a cell type that in mice is found exclusively in the basal plate, first in the VZ and then in the MZ, suggesting that Rgl2 migrate away from the VZ during VM development. Human and mouse Rgl2 share the expression of several gliogenic markers, such as the glia specific glutamate transporter Slc1a3 (Torp et al., 1994; Lehre et al., 1995) and the astrocyte markers Aldoc (Walther et al., 1998) and Tnc (Karus et al., 2011), suggesting that Rgl2 cells in both species may play a role in gliogenesis. Interestingly, three molecularly defined Rgl2 subtypes (Rgl2a-c) were identified in the human VM based on their expression profile, indicating that they may serve some more specialized functions in human midbrain development. It is interesting to note that the transcriptional profile of $\mathrm{Rgl} 2 \mathrm{c}$ was found to be similar to that of oligodendrocyte precursor cells (OPCs), with these two cell types sharing the expression of Olig2, an important OPC marker (Fu et al., 2002). Since Rgl2c is detected at human embryonic week 10 and OPCs are detected around weeks $10-11$, we suggest that $\mathrm{Rgl} 2 \mathrm{c}$ may be the cell type giving rise to OPCs. Lineage-tracing experiments should be performed in the future to determine whether there is any lineage relation between these two cell types.

The third radial glia cell type, $\mathrm{Rgl} 3$, is found predominantly in the mouse floor plate. Both mouse and human Rgl3 are characterized by the expression of several secreted factors implicated in different aspects of midbrain development. These include Slit1, Slit2, and Ntn1, involved in neuronal migration and axon guidance (Livesey and Hunt, 1997; Lin et al., 2005), Spon1 (Klar et al., 1992), as well as morphogens and growth factors of the transforming growth factor-beta (TGFB) family (Poulsen et al., 1994; Arenas et al., 2015b).

These data suggest that during weeks $6-11$ of human embryonic development, Rgl3 may serve the classical role of floor plate radial glia as a signaling center, while Rgl1 could play a role as neurogenic radial glia and Rgl2 in the basal plate could be specialized in gliogenesis. Future studies should focus on addressing the current gaps in our knowledge regarding the role of radial glia in human development. These should include determining the position that human radial glia occupy along the ventral-dorsal and anterior-posterior axis of the VM VZ, 

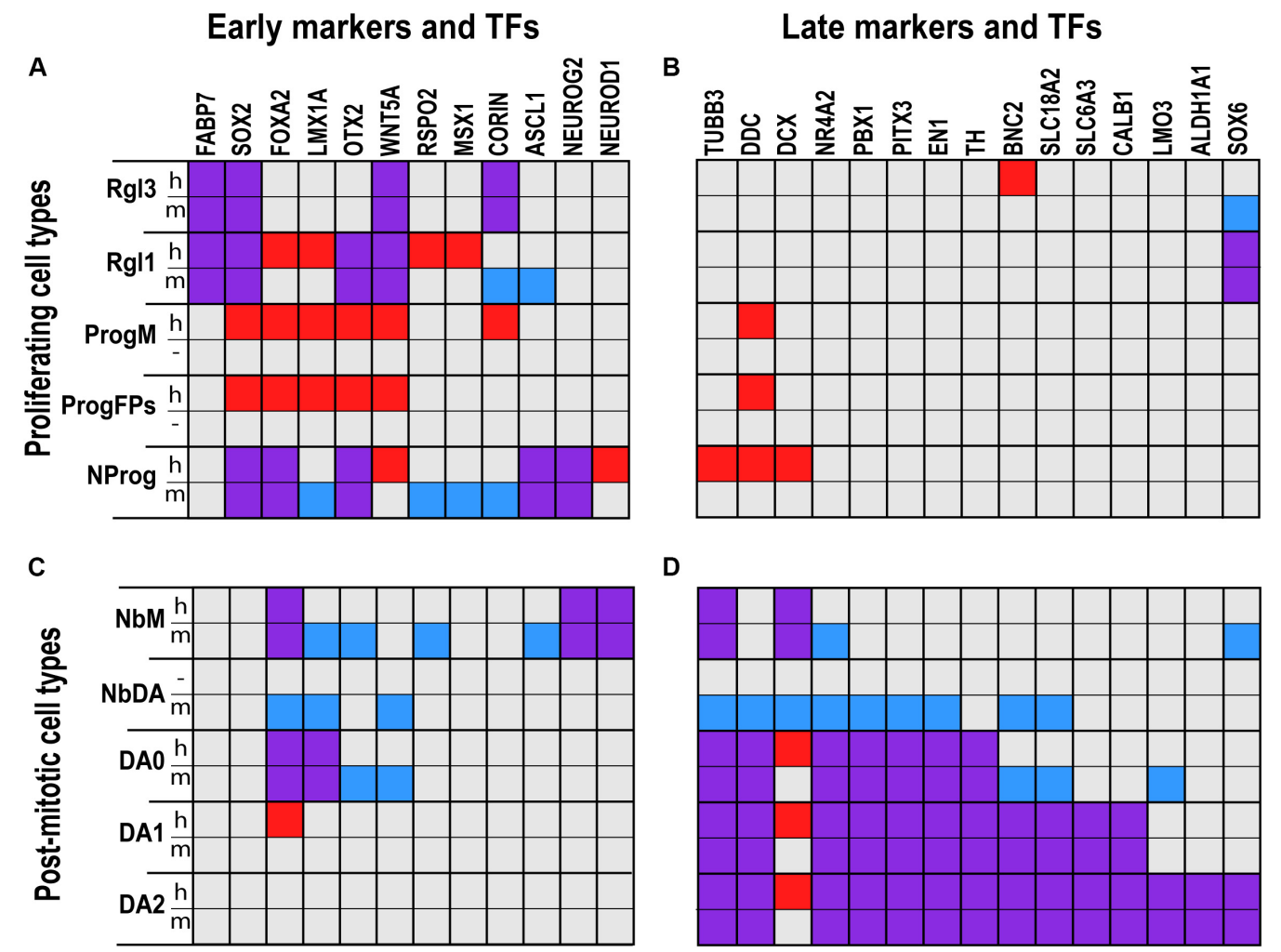

FIGURE 1 | Comparative analysis of gene expression in defined cell types of the developing human and murine ventral midbrain as assessed by scRNA-seq. Proliferating $\mathbf{( A , B )}$ and post-mitotic cell types $\mathbf{( C , D )}$ expressed distinct early $\mathbf{( A , C )}$ and late $\mathbf{( B , D )}$ marker genes and transcription factors (TFs). Squares in red denote genes detected only in human; in blue, detected only in mouse; and in purple, detected in both human and mouse. Gene expression was more similar across species in radial glia (Rgl1, 3) and dopaminergic neurons (DA0-2) than in neuronal progenitors (NProg) or neuroblast medial (NbM). Cells such as medial progenitors (ProgM) or lateral and medial floorplate progenitors (ProgFPs) were only found in human, whereas the dopaminergic neuroblast (NbDA) was only found in mouse. Cell type and gene expression from La Manno et al. (2016).

and whether radial glia cells can migrate to the IZ and $\mathrm{MZ}$, as suggested by smFISH analysis of the mouse VM (La Manno et al., 2016). Finally, the precise function and contribution of each radial glia cell type to human $\mathrm{mDA}$ neuron development remains to be determined, e.g., through experimental ablation of these cell types.

\section{Progenitor Cell Types in the Developing Human Midbrain}

scRNA-seq analysis of the developing human midbrain also revealed a great diversity of progenitor cell types (La Manno et al., 2016), with molecular identities that largely correspond to previously described progenitor domains in the mouse and human VM (Andersson et al., 2006b; Prakash et al., 2006; Ono et al., 2007; Hebsgaard et al., 2009). The human VZ was found to contain five distinct progenitor cell types, which were named based on their molecular identity as well as their presumed location and/or function in the $\mathrm{VZ}$ (ProgM [midline progenitor]; ProgFPL [lateral floor plate progenitor], ProgFPM [medial floor plate progenitor]; ProgBP [basal plate progenitor]; NProg [neuronal progenitor]) (La Manno et al., 2016). However, it should be noted that these human progenitor cell types were named after the similarity of their transcriptomes to that of their homologous mouse cell types, but not because their position in the human VM, which still remains to be determined. It is thus possible that some of the human progenitor cell types may occupy a different or unsuspected position in the human VM. A systematic histological identification of the position of human progenitor cell types in the developing human VM will be necessary to define their spatial organization.

Human progenitor cells were found to share some characteristics with radial glia cells, such as the expression of the neural stem cell marker SOX2 (Graham et al., 2003), and the expression of RFX4, a TF that modulates Shh signaling (Ashique et al., 2009). However, they also have specific characteristics that set them apart from radial glia cells, e.g., they all express the chromatin remodeling proteins HMGA1 and HMGB2 (Hock et al., 2007). Additionally, each progenitor is characterized by a unique molecular signature, discussed below.

The midline progenitor (ProgM) is defined by the expression of CORIN (Figure 1A), a natriuretic peptide-converting enzyme that marks the murine VM midline (Ono et al., 2007), as well as TOX, a transcriptional regulator of SOX2 (Artegiani et al., 2015), and $J A D E-1$, an inhibitor of the Wnt signaling pathway 
(Chitalia et al., 2008). Both floor plate progenitors (ProgFPL and ProgFPM) are characterized by the expression of LMX1A, a transcription factor that specifies $\mathrm{mDA}$ neurons and labels the human floor plate (Figure 1A) (Andersson et al., 2006b; Hebsgaard et al., 2009; Nelander et al., 2009). Additionally, the lateral floor plate progenitor (ProgFPL) is identified by a high expression of the morphogen WNT1, which is required for midbrain and $\mathrm{mDA}$ neuron development in mice (McMahon and Bradley, 1990; Thomas and Capecchi, 1990; Danielian and McMahon, 1996; Prakash et al., 2006; Andersson et al., 2013; Yang et al., 2013), and ZEB2, a transcription factor that regulates $\mathrm{mDA}$ progenitor proliferation and neurogenesis (Yang et al., 2018). Conversely, the medial floor plate progenitor (ProgFPM) is characterized by low WNT1 expression. Basal plate progenitors (ProgBP) were identified by the expression of FOXA2 in the absence of the floor plate markers mentioned above, such as WNT1 and LMX1A. The final progenitor identified in the human VZ, NProg, is not characterized by the expression of genes in a defined medial-lateral compartment, but rather by the expression of pro-neural genes such as NEUROG2 and ASCL1 (Andersson et al., 2006a; Kele et al., 2006); genes known to regulate $\mathrm{mDA}$ neurogenesis such as OTX2 (Omodei et al., 2008) (Figure 1A), and early neuronal markers such as TUBB3 (Figure 1B). We thus hypothesize that this human progenitor reflects a class of neurogenic progenitors capable of giving rise to post-mitotic neuroblasts and neurons. A similar NProg was also identified in the mouse developing midbrain. In addition to Neurog2 and Ascl1, the mouse NProg also express genes found in the human ProgM and ProgFPs, such as Corin and Lmxla, respectively (Figure 1A). This observation and the fact that proliferative progenitors with distinct mediolateral identities were not identified in the mouse suggests that mouse progenitors identified at E11.5 are in a more differentiated neurogenic state compared to human week 6 . Further analyses will be needed to determine whether the progenitor diversity found in the human VM can be identified at earlier stages in mice and whether such diversity may also lead to further subdivisions of the NProg cluster if ProgBP, ProgFPL, ProgFPM, or ProgM progenitors retain their compartment identity while undergoing neurogenesis.

\section{Human VM Post-mitotic Cells}

In addition to the variety of progenitor cell types detected in the VZ, our scRNA-seq analysis also revealed a great diversity of post-mitotic cell types in the developing human and murine VM. Post-mitotic cells in the developing VM were commonly characterized by a loss of proliferative markers and the gain of markers such as TUBB3 ( $\beta$-III-tubulin), an early cytoskeletal neuronal marker (Jiang and Oblinger, 1992), and SNAP25, a late synaptic marker (Söllner et al., 1993). Post-mitotic cells can be further divided into neuroblasts and neurons. Neuroblasts are immature migratory cells born from progenitor cells that progressively mature and differentiate into neurons, the cell types expressing all the machinery required for the synthesis, release, and uptake of neurotransmitters. While all neuroblast cell types share some common features, they can be distinguished by the differential expression of transcription factors that define their lineage and spatial position. Comparison of mouse and human single-cell transcriptomes revealed the presence of three common neuroblast cell types in the mouse and human. These cells were named based on their presumed location in the developing mouse VM ( $\mathrm{NbM}$ [neuroblast medial], NbML1 and 5 [neuroblast medio-lateral]), with NbM and NbML1 being the earliest appearing and the most abundant neuroblasts in the developing VM of both species (La Manno et al., 2016).

In both human and mouse, $\mathrm{NbM}$ was characterized by the expression of several bHLH factors such as NEUROD1 and NEUROG2 (Figure 1C), which play essential roles in neurogenesis and differentiation (Kele et al., 2006; Dennis et al., 2019). Additionally, mouse NbM was found to express Nr4a2 (Nurr1) (Figure 1D), a nuclear receptor required for mDA neuron development (Zetterström et al., 1997). While expression levels of NR4A2 were lower in human $\mathrm{NbM}$, histological analysis of the developing human $\mathrm{VM}$ has shown that NR4A2 is present in tyrosine hydroxylase-negative $\left(\mathrm{TH}^{-}\right)$neuroblasts in the floor plate and $\mathrm{TH}^{+} \mathrm{mDA}$ neurons in the VM (Marklund et al., 2014). The same expression pattern of Nr4a2 can also be seen in histological analysis of the mouse developing midbrain (Villaescusa et al., 2016), indicating that in both the human and mouse VM, $\mathrm{NbM}$ differentiate into $\mathrm{mDA}$ neurons. Additionally, Nr4a2 was also expressed in a more differentiated mouse $\mathrm{mDA}$ neuroblast (NbDA) (Figure 1D), a cell type which was identified by scRNA-seq in mouse but not in the human VM (La Manno et al., 2016).

The second most abundant neuroblast in human and mouse is the mediolateral neuroblast 1 (NbML1), which is characterized by the expression of the transcription factor Nkx6-2 (La Manno et al., 2016). In the mouse, NbML1 cells are defined by the expression of Nkx6-2 and Cartpt (cocaine- and amphetamine-regulated transcript prepropeptide), two markers labeling in the IZ/MZ of the basal plate (La Manno et al., 2016). Cart is transiently expressed in an embryonic population in the $\mathrm{MZ}$ of the presumptive dorsal tegmentum, as well as in the embryonic and adult Edinger-Westphal Nucleus in the rat (Brischoux et al., 2002). In the mouse, Cartpt is expressed by several postnatal neurons in the dorsal-medial tegmentum, including the Edinger-Westphal nucleus, periaqueductal gray, oculomotor and trochlear neurons (Allen brain atlas, 2004, image 18). In addition, $15 \%$ of the red nucleus (RN) neurons in mice express Nkx6-2 (Prakash et al., 2009; Moreno-Bravo et al., 2010). We thus suggest that NbML1 cells may give rise to different Cartpt+ or Nkx6-2+ neuron types that will occupy the dorsal-medial tegmentum. Ultimately, spatial transcriptomics and lineage tracing experiments will be, respectively, required to determine the position and cell types generated by NbML1 in mouse and human.

Besides neuroblasts, several homologous neuronal cell types were detected in the developing human and murine VM, including dopaminergic; serotonergic; GABAergic; oculomotor and trochlear nucleus (OMTN) and RN neurons. All the neuronal cell types were identified based on the expression of components necessary for neurotransmission. 
This included the rate-limiting enzyme for neurotransmitter synthesis, the vesicular transporter responsible for loading the neurotransmitter into synaptic vesicles as well as the neurotransmitter reuptake transporters. In the case of $\mathrm{mDA}$ neurons, they were characterized by the expression of $\mathrm{TH}$, the rate-limiting enzyme in dopamine synthesis, SLC18A2 (VMAT2), and SLC6A3 $(D A T)$. However, three distinct types of embryonic mDA neurons could be detected in the developing human and murine VM (DA0-2) (Figure 1D).

The earliest embryonic mDA neuron, DA0, was detected at week 6 (E11.5 in mouse). This cell type expresses $T H$ but lacks SLC6A3, and thus it was classified as an incomplete DA neuron not yet capable of neurotransmission. At week 7 (E12.5 in mouse), the first mDA neuron cell type (DA1) that expresses SLC6A3, thus fulfilling all the criteria for neurotransmission, was detected. A second mDA neuron (DA2) was detected at week 8 (E13.5 in mouse) and was found to additionally express SOX6, a transcription factor (Panman et al., 2014), LMO3, a transcriptional co-activator of Pitx3 (Bifsha et al., 2017) and aldehyde dehydrogenase 1 family member A1 (ALDH1A1), an enzyme involved in DA catabolism (Goldstein et al., 2013) as well as the synthesis of retinoic acid (McCaffery and Drager, 1994) and GABA (Kim et al., 2015). In the adult murine midbrain, the expression of these three genes overlap only in the ventral tier of the $\mathrm{SNc}$ pars compacta. Indeed, mDA neurons expressing Aldh1a1 are located in the ventral part of the ventral tegmental area (VTA), and the ventral tier of the SNc pars compacta (Galter et al., 2003; Poulin et al., 2018). Sox6 expressing mDA neurons are found in both the ventral and dorsal tier of the $\mathrm{SNc}$ and the dorsolateral VTA (Panman et al., 2014; Poulin et al., 2018). Lmo3 is preferentially expressed in SNc mDA neurons (Bifsha et al., 2017). These results suggest that embryonic mouse and human DA2 neurons are likely candidates to give rise to $\mathrm{mDA}$ neurons of the SNc. However, the molecular diversity of the adult human midbrain and postnatal $\mathrm{mDA}$ neurons remains to be elucidated at the single-cell level. Future studies should aim to characterize this diversity and to understand the lineage relationship between embryonic DA neurons and postnatal/adult mDA neuron subtypes.

\section{TEMPORAL DIFFERENCES BETWEEN HOMOLOGOUS CELL TYPES IN HUMAN AND MOUSE DEVELOPMENT}

To determine potential differences in temporal dynamics between human and mouse midbrain development, investigators have traditionally relied on histological analysis and examined specific markers that define cell types at different developmental time-points. For instance, histological analysis of the human embryonic midbrain has revealed that human mDA neurons, identified by $T H$ expression, appear at weeks 5-6 post-conception, mDA neurogenesis peaks around w7-8 and then ceases by w10-11 (Freeman et al., 1991; Almqvist et al., 1996; Puelles and Verney, 1998; Verney et al., 2001), resulting in $\sim 400.000-600.000$ human $\mathrm{mDA}$ neurons
(German et al., 1983; Pakkenberg et al., 1991). In contrast, murine mDA neurogenesis takes place in only 6 days, with neurogenesis beginning at E9.5, peaking at E11.5 and then ceasing at around E14.5-15.5 (Bayer et al., 1995), resulting in $~ 30.000$ murine mDA neurons (Nelson et al., 1996). The significant increase in gestation time for humans would be expected to result in an even greater number of $\mathrm{mDA}$ neurons. However, cell cycle length is longer in human progenitors, resulting in neurogenesis not being directly proportional to the increase in gestation time (Kornack and Rakic, 1998). Indeed, it has been estimated that human progenitors in the $\mathrm{VZ}$ are only about half as proliferative as those in the mouse (La Manno et al., 2016).

However, to compare developmental time across species in a more precise manner, it is important to consider not one, but a series of neurodevelopmental events. To this end, Workman et al. (2013) developed a generalized linear regression model, which correlates brain development across different species based on a series of anatomical and developmental milestones. This model will be used here to compare the temporal axis of homologous cell types in the developing VM to determine whether differences in temporal dynamics exist between human and mouse midbrain development.

Based on the model by Workman et al. (2013), the human embryonic midbrain at w6 is homologous to mouse E11.5. After that, one week of human midbrain development is less than one embryonic day in the mouse, with w11 being homologous to E15.5 (Figure 2A). However, it is important to keep in mind that while such models allow for comparison between human and mouse midbrain development, it is unclear whether there is a perfect temporal correlation between homologous cell types across the two species. Indeed, the time at which a human or murine homologous midbrain cell type is first detected by scRNA-seq, or the time at which $50 \%$ of a certain cell type has appeared (average time of appearance) varies considerably ( $\mathrm{La}$ Manno et al., 2016) even if equivalent periods of time in human (w6-w11) and mouse (E11.5-15.5) development (according to Workman) are considered.

Temporal differences between mouse and human VM development can already be detected in proliferating cells at early developmental stages, as illustrated by the different order of appearance of VM radial glia cell types. Murine Rgl1 cells are detected at $\leq \mathrm{E} 11.5$, while $\mathrm{mRgl} 2$ and $\mathrm{mRgl} 3$ emerge at E12.5, as assessed by scRNA-seq and smFISH. Conversely, in the developing human midbrain, the earliest radial glia cell type detected by scRNA-seq is hRgl3, appearing at w6 [homologous mouse time (hmt) of E11.5], a day earlier than mRgl3 (E12.5). On the other hand, hRgl1 appears at w7 (hmt $\approx$ E12.5), a day later than mRgl1 (E11.5). Finally, human Rgl2a-b appear at w7 (hmt $\approx$ E12.5, a similar developmental stage to the mouse), while $\mathrm{hRgl} 2 \mathrm{c}$ is only found at $\mathrm{w} 10$ (hmt $\approx$ E15, i.e., 2.5 days after $\mathrm{mRgl} 2$ is detected) (Figure 2B).

Similar species differences are also detected when considering the average time of appearance for each radial glia, although they are less clear (Figure 2C). In human, Rgl1 is found on average slightly later than mouse Rgl1 (w9, hmt $\approx$ E14 vs. E13.5), 

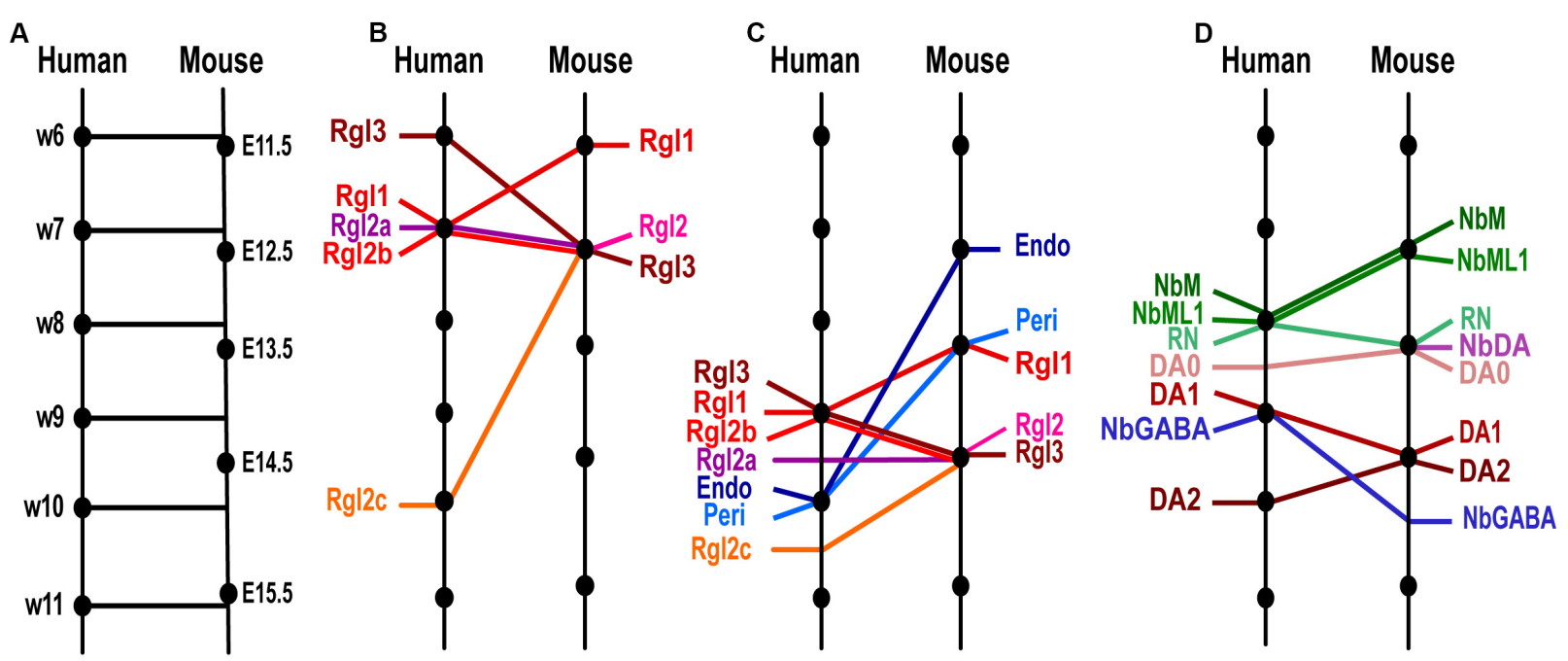

FIGURE 2 | Temporal differences between homologous cell types in human and murine ventral midbrain development as detected by scRNA-seq. (A) Model of the time equivalence between human and murine midbrain development, as defined by Workman et al. (2013) based on developmental features. Homologous time-points are indicated with lines perpendicular to the time-line. (B) Comparison of the time of appearance of human and mouse Radial glia (Rgl) cell types, showing significant species differences (oblique lines) in Rgl3, Rgl1 and Rgl2c. (C) Progenitor and non-neuronal cell types ordered by average time of appearance as detected by scRNA-seq. Note that vascular cells, endothelial (Endo) and pericytes (Peri) appear earlier in mouse than in human and show greater temporal differences than Rgl types. (D) Post-mitotic cell types ordered by average time of appearance showing greater temporal species differences in neuroblasts than in neurons. Cell type and time-line from La Manno et al. (2016).

while human $\mathrm{Rgl3}$ is found slightly earlier (w9, hmt $\approx \mathrm{E} 14$ ) than mouse Rgl3 (E14.5). However, human Rgl2 are found at similar stages as $\mathrm{mRgl} 2$ (E14.5), with hRgl2b found at w9 (hmt $\approx \mathrm{E} 14), \mathrm{hRgl} 2 \mathrm{a}$ at $\mathrm{w} 9.5$ (hmt $\approx \mathrm{E} 14.5)$, and hRgl2c at w10.5 $(\mathrm{hmt} \approx \mathrm{E} 15)$.

These results, as well as species-specific differences identified in the transcriptional profile of the different $\mathrm{Rgl}$ cells, discussed below, raise the interesting question of whether human and murine $\mathrm{Rgl}$ cell types serve entirely homologous or some species-specific functions in midbrain development. Gain and loss of function experiments will be required to determine their individual function and how changes in their temporal order of appearance could affect midbrain development. An additional critical issue that also remains to be addressed is the lineage relationship between distinct radial glia subtypes and their relationship to the diverse progenitor cell types in the midbrain.

Temporal differences between human and mouse midbrain development were also found in post-mitotic cell types. For instance, the two most abundant midbrain neuroblast cell types, the medial neuroblast $(\mathrm{NbM})$ and the mediolateral neuroblast type 1 (NbML1), are both found at later stages in human development compared to the mouse. Indeed, the average time at which both cell types are detected in the mouse is E12.5, while their human counterparts are detected on average at w8 (hmt $\approx$ E13.5, i.e., 1 day later). Other neuroblasts that appear later in development, such as NbGaba, are also detected at non-homologous developmental times, but in this case, they are detected on average at earlier stages in human (w9, hmt $\approx \mathrm{E} 14$ ), compared to mouse (E15) (Figure 2D). These results indicate that homologous neuroblasts can be found at non-homologous time-points, suggesting that each neuroblast has a unique developmental timeline in each species.

\section{DIFFERENCES IN THE DOPAMINERGIC LINEAGE AND THE TIME SEQUENCE AT WHICH CELL TYPES EMERGE DURING HUMAN AND MOUSE DEVELOPMENT}

Lineage tracing studies in the mouse have demonstrated that $\mathrm{mDA}$ neurons are derived from progenitors that express morphogens such as Shh and Wnt1 (Zervas et al., 2004; Joksimovic et al., 2009; Blaess et al., 2011; Brown et al., 2011), as well as the glutamate astrocyte transporter Slc1a3 (Glast) (Bonilla et al., 2008) and the floor plate marker Corin (Ono et al., 2007). Shh expression in the VM floor plate is dynamic and has been shown to delineate different progenitor domains, including progenitor domains that will later give rise to DA, RN and OMTN neurons. All mDA neurons appear to arise from VM floor plate progenitors that express Shh and respond to high levels of Shh signaling (as assessed by Gli1 expression), with mDA progenitors expressing Shh between E8.5-11.5 and responding to Shh signaling between E7.5-E9.5. Shh expressing progenitors contribute the most significant amount of $\mathrm{mDA}$ neurons at E9.5, at the time when Shh expression is detected in the entire Lmxla domain of the FP (Joksimovic et al., 2009; Blaess et al., 2011). This indicates that mDA neurons are derived from progenitors that co-express Shh and Lmxla. Conversely, at E11.5, when Shh is downregulated in the floor plate midline, Shh expressing progenitors no longer contribute 
mDA neurons (Joksimovic et al., 2009; Blaess et al., 2011). Additionally, fate-mapping studies show that progenitors in the midbrain-hindbrain boundary and VM floor plate expressing Wnt1 between E7.5-13-5 also generate mDA neurons (Zervas et al., 2004; Prakash et al., 2006; Brown et al., 2011), particularly at E9.5 (Brown et al., 2011). Combined, all the results described above indicate that progenitors in the VM hindbrain boundary and the midbrain floor plate give rise to $\mathrm{mDA}$ neurons.

These fate-mapping studies were done at an early developmental time point, making them difficult to interpret in the context of our scRNA-seq data. However, mDA neurons have also been fate mapped from radial glia cells at E10.5-11.5 using the glutamate astrocyte transporter Slc1a3 (Bonilla et al., 2008), which is expressed in all radial glia cells in the murine VM (mRgl1-3) (La Manno et al., 2016). Furthermore, mDA neurons can be derived from VM progenitors sorted at E13.5 for the midline marker Corin (Ono et al., 2007), which is expressed in mRgl1/3 and NProg (La Manno et al., 2016). Thus, comparing scRNA-seq data with previous lineage tracing and FACS sorting suggests that mDA neurons may be derived from mRgl1. However, $\operatorname{Lmx} 1 a$, a gene required for $\mathrm{mDA}$ neuron specification (Andersson et al., 2006b), is not expressed in mRgl1, but rather in $\mathrm{mNProg}$ and downstream cells in the mDA lineage: $\mathrm{mNbM}$, mNbDA and mDA0 (Figure 1). Additionally, our scRNA-seq data shows that mRgl1, as well as mNProg and mNbM, express Ascl1 (marking the onset of neurogenesis), mNProg and $\mathrm{mNbM}$ express Neurog2 (a gene required for mDA neurogenesis), while mNbM express NeuroD1 (a gene downstream in neurogenesis). This suggests a sequence of events in which mRgll becomes $\mathrm{mNProg}$, which then gives rise to the first post-mitotic cell, mNbM. Finally, histological and knockout analysis in mice suggest that Neurog2+ cells in the VZ (mNProg) give rise to $\mathrm{Nr} 4 a 2+$ cells in the IZ (mNbM) (Kele et al., 2006), which in turn subsequently differentiate into $P b x 1+\mathrm{mNbDA}$ and Th+ DA neurons (Villaescusa et al., 2016), with the embryonic DA neurons appearing sequentially, from mDA0 to mDA2 (La Manno et al., 2016).

Much less is known about the lineages in the developing human midbrain. However, recent studies in which human embryonic stem cells (hESCs) have been FACS sorted during mDA neuron differentiation have provided some interesting information. Antibodies targeting proteins such as CORIN (Doi et al., 2014), ALCAM (Bye et al., 2015) or CD47 (IAP) (Lehnen et al., 2017), all of which are expressed by hRgl3 and hProgM (La Manno et al., 2016), have been used to isolate progenitors capable of giving rise to human $\mathrm{mDA}$ neuroblasts $(\mathrm{hNbM})$ and $\mathrm{mDA}$ neurons (hDA0-2). Similarly, $L M X 1 A$, a gene expressed in hRgl1, hProgM, hProgFPM, and hProgFPL (La Manno et al., 2016), has been used to generate hESC reporter lines that, after sorting, can generate human mDA neuroblasts and neurons (Samata et al., 2016; Niclis et al., 2017). These results suggest that in humans, the first cell type capable of mDA neurogenesis is hProgM at week 6 (instead of mRgll in mice). As in the mouse, the next candidate cell type in the DA lineage is hNProg, which expresses several genes involved in mDA neurogenesis (Ascl1, Neurog2, and NeuroD1), followed by hNbM, which expresses Neurog2 and NeuroD1. However, no human NbDA was detected and the next cell type detected in the $\mathrm{mDA}$ lineage is hDA0, followed by hDA1 and then hDA2 (Figure 2D), which progressively acquire the expression of mDA neuron subtype markers (Figure 1D).

The results above suggest important species differences in mDA neuron development, such as the first candidate cell type in the human mDA lineage being a non-homologous cell type (hProgM vs. mRgl1), that NbDA is either not found or is a very transient population in humans (see discussion below), and that gene expression in any given cell type of the human mDA lineage is not identical to that in the homologous mouse cell type (Figure 1A). Ultimately, deeper and more extensive scRNA-seq and spatial transcriptomics analysis will improve the definition of these cell types, their expression profile and their positions within the developing midbrain. Moreover, lineage tracing experiments using molecular barcodes and single-cell sequencing methods in human tissue or stem cell preparations (2D or $3 \mathrm{D})$ will be needed to decipher the actual lineage relationship within the mDA lineage and define the kinetics of cell-to-cell transitions during human development.

\section{TEMPORAL DIFFERENCES IN LINEAGE TRANSITIONS BETWEEN HUMAN AND MOUSE DEVELOPMENT}

Using the model developed by Workman et al. (2013), the time at which each cell type appears according to the scRNA-seq analysis and the limited lineage information available, it is also possible to estimate how long it takes for a given post-mitotic neuroblast to generate a downstream neuron type. In this way, we find that mouse $\mathrm{NbM}$ cells take 2 days to generate DA1 neurons (mNbM found at E12.5 and mDA1 at E14.5), whereas human NbM cells generate DA1 in less than 1 week (w8-8.75), corresponding to less than a day in homologous mouse time (hmt) (Figure 2D). The short time it takes for hNbM cells to generate hDAl neurons suggests that intermediate cell types in the DA lineage may only exist for a very brief period of time, making them difficult to detect. In support of this hypothesis, we reported that $\mathrm{NbDA}$, a cell type directly downstream of NbM in the mouse (Villaescusa et al., 2016), was not detected in the human midbrain, as it may exist only for a very brief time in human development (La Manno et al., 2016). Further scRNA-seq analysis of the developing human VM will be needed to determine whether this is the case.

Notably, the species difference in the kinetics for generating hDA1 neurons seems to be cell-type specific because other cell populations do not follow the same dynamics. For instance, NbML1 neuroblasts, which are thought to give rise to $15 \%$ of red nucleus (RN) neurons, take comparable homologous developmental time in both species; less than a day in the mouse and within half a week in the human (Figure 2D). Thus, currently available data indicate that VM neuroblasts generate neurons following both cell type- and species-specific temporal kinetics.

In addition to neural cells, the cells that make up the vasculature in the midbrain, such as pericytes and endothelial cells, appear relatively late in human development compared to the mouse. While human endothelial cells and pericytes are detected on average at w10 (hmt $\approx \mathrm{E} 15)$, they are detected 
significantly earlier in the mouse, at E13 and E14, respectively (Figure 2C). This finding is somewhat surprising, as a slower vascularization of the human VM would limit oxygen and nutrient supply compared to the mouse midbrain. These conditions may favor the maintenance of cells with anaerobic metabolism such as progenitor cell types and slow down the maturation of neurons in the human VM.

In sum, from this analysis, it becomes apparent that while some cell types are generated at homologous developmental time points, following the model by Workman et al. (2013), others are not. These results suggest that cells do not follow a universal homologous linear timeline across species, but instead follow their own cell type- and species-specific developmental time. This finding may have important implications as cells may become functional at different developmental times, resulting in species-specific cell type assemblies and interactions at the tissue level. Future experiments should aim to elucidate how these observed temporal differences in cell types across species may impact the regulation of gene expression, cell signaling, and metabolism within the tissue. Furthermore, it remains to be determined how such temporal differences may impact key developmental events such as lineage progression, migration, and functional maturation in a species-specific manner.

\section{DIFFERENCES IN TRANSCRIPTION FACTOR EXPRESSION BETWEEN HUMAN AND MOUSE CELL TYPES DURING MIDBRAIN DEVELOPMENT}

The function of transcription factors in $\mathrm{mDA}$ neuron development has been extensively studied in the mouse (reviewed in Arenas et al., 2015a; Blaess and Ang, 2015; Bodea and Blaess, 2015; Smidt, 2017). However, their function in human mDA neuron development remains largely unknown. Our scRNA-seq analysis and comparison of the developing human and murine VM demonstrated that TFs required for mDA neuron development in mice are generally expressed in homologous mouse and human cell types (La Manno et al., 2016). These results suggest that most TFs will form similar transcriptional networks and may serve similar functions in homologous cell types in both species. Nonetheless, key TFs commonly used to identify cells in the mDA lineage are also expressed in non-homologous cell types. In previous sections, we described how homologous cell types in human and mouse differ in gene expression profiles. Here, we examine how the choice of common TFs to identify cell types may impact the identification of cell types both within one species and when comparing across species. In interpreting this data, it should be noted that TFs are usually expressed at low levels and are thus difficult to detect, which may lead to false negative results. It should also be noted that the developmental time at which cells are sampled and the number of cells sampled may have influenced the results to some extent. It thus remains to be determined whether by analyzing additional developmental stages and a greater number of cells with improved sequencing methods, it may be possible to detect additional cell types and differences in gene expression in a more reliable manner. Undoubtedly, as the methodology and data analysis improve, we can in the near future expect to gain a better definition of the cell types and genes that control mDA neuron development.

\section{Factors Specifying mDA Progenitors}

As discussed above, disparities in TF expression between homologous murine and human cell types are already apparent at early developmental stages. Notably, differences in TF expression, such as $L M X 1 A$ in hRgl1 or mNProg, are not isolated events, but rather reflect more profound changes in TF expression signatures, indicating that non-homologous cell types share surprisingly large TF expression signatures. For instance, in the developing human midbrain, hRgl1 expresses several of the TFs that identify mDA progenitors, such as SOX2, OTX2 (Vernay et al., 2005), FOXA2 (Ferri et al., 2007), LMX1A, and MSX1 (Andersson et al., 2006b). However, in the mouse, the combination of these factors is not expressed in mRgll, but rather in a non-homologous cell type: the neuronal progenitor (mNProg) (Figure 1A). This observation is both interesting and unexpected because several of these TFs are known to regulate each other and are part of a developmental pathway that controls $\mathrm{mDA}$ progenitor identity and the generation of $\mathrm{mDA}$ neurons. However, these two cell types, in keeping with their identification as non-homologous cell types, differ in the expression of other TFs. For instance, hRgl1 expresses $L H X 3$, which is involved in motor neuron specification (Sharma et al., 1998), while $\mathrm{mNProg}$ expresses TFs controlling mDA neurogenesis, such as Ascl1 and Neurog2 (Kele et al., 2006). Thus, the expression of these additional TFs may lead to the formation of distinct transcriptional networks, expression profiles, and perhaps even additional functions such as motor neuron development in the case of hRgl1.

Similar observations can be made when different cell types from the same species are surveyed with a combination of markers. For instance, OTX2, FOXA2, LMX1A, and WNT5A are commonly expressed in distinct human proliferating cell types such as hRgll, hProgM hProgFPM, and hProgFPL (La Manno et al., 2016), which are distinguished from one another by the expression of additional TFs and morphogens that may confer different functions. Thus, commonly used combinations of genes currently thought to identify one cell type can, in fact, identify either multiple cell types within one species or even non-homologous cell types across species. These results emphasize the importance of examining broad marker panels supported by single-cell data or the use of single-cell transcriptomic profiling to identify the correct cell type.

Another interesting finding is that markers such as OTX2, FOXA2, LMX1A, and WNT5A, which are used to define generic mDA neuron progenitors, are expressed at significant levels in most early human proliferating cell types (hRgl1, hProgM, hProgFPM, and hPRogFPL). Notably, their expression then rapidly decreases in hNProg and is low during differentiation in human cell types, but not in the mouse. Indeed, unlike mNProg, hNProg expresses significant levels of only two of these four factors: FOXA2 and WNT5A (Figure 1A). Moreover, no human 
post-mitotic cell type expresses significant levels of more than two of these factors, while several mouse post-mitotic cell types do, including mNbM (Foxa2, Lmx1a, and Otx2), mNbDA (Foxa2, Lmx1a, and Wnt5a), and mDA0 (Foxa2, Lmx1a, Otx2, and $W n t 5 a)$. Indeed, the only human post-mitotic cell type expressing more than one of these factors is hDA0 (FOXA2 and LMX1A) (Figure 1C) (La Manno et al., 2016). These results suggest that TFs may be more difficult to detect in human tissue and/or that human post-mitotic cell types may not require the expression of as many or as high expression levels of early genes compared to their homologous mouse cell types. Future experiments should aim to distinguish between these possibilities.

\section{Factors Controlling mDA Neurogenesis}

In the mouse, $\mathrm{mDA}$ neurogenesis is controlled by the pro-neural basic helix-loop-helix TF Neurog2 (Andersson et al., 2006a; Kele et al., 2006). This TF is expressed in homologous human and mouse NProg and NbM (Figures 1A,B), indicating that neurogenesis occurs in the same cell type in both species. In agreement with this, other genes involved in neurogenesis are also expressed in these homologous cell types, such as ASCL1 (in NProg), or NEUROD1 (in NbM). Additionally, in both species, NProg cells share the expression of SOX2 and FOXA2 (Figure 1A), whereas $\mathrm{NbM}$ share the expression of FOXA2, TUBB3, and DCX (Figures 1C,D). Nevertheless, the expression of other TFs that regulate $\mathrm{mDA}$ neuron development differs greatly in the two homologous cell types. While the mouse NProg express several early genes such as $\operatorname{Lm} x 1 a$, Otx2, Rspo2, Msx 1, and Corin (Figure 1A), human NProg express later stage neuronal genes found in $\mathrm{mNbM}$ such as NEUROD1, TUBB3, and DCX, or even in mNbDA such as DDC (DOPA decarboxylase) (Figure 1). Similarly, mouse NbM express more early genes than human $\mathrm{NbM}$, such as Lmx1a, Otx2, Rspo2, and Ascl1 (Figure 1C). These results suggest that mouse NProg and NbM not only express earlier developmental genes, but they might be at an earlier developmental stage than human NProg and NbM. In agreement with this, our global transcriptomic analysis of the temporal differences between mouse and human NbM (Figure 2D) also suggests that mouse NbM cells are at an earlier homologous developmental time compared to human NbM.

\section{Factors Expressed in Post-mitotic mDA Cell Types}

At first glance, the genes that define post-mitotic cell types in the mDA lineage are largely conserved in homologous cell types, but several differences can also be observed (Figures 1C,D). Expression of the nuclear receptor $\mathrm{Nr} 4 \mathrm{a} 2$ in post-mitotic neuroblasts is required for the acquisition and maintenance of the mDA phenotype (Zetterström et al., 1997; Castillo et al., 1998; Saucedo-Cardenas et al., 1998; Kadkhodaei et al., 2009). In the mouse, $\mathrm{Nr} 4 \mathrm{a} 2$ is expressed at high levels in $\mathrm{NbM}$ cells and is maintained in all cell types of the mDA lineage (NbDA; DA0-2), whereas human NR4A2 is only expressed at high levels in embryonic mDA neurons (DA0-2), suggesting that differences in gene regulation exist between mouse and human. Additionally, immunocytochemical analysis of fetal human VM tissue has indicated that NR4A2 protein is present in cells that have not yet acquired the expression of $T H$, pointing toward the existence of human DA neuroblasts (NbDA) that express NR4A2 (Villaescusa et al., 2016), which were not detected by scRNA-seq (La Manno et al., 2016). These differences in transcript and protein detection may be explained by differential regulation of transcription and translation as well as differences in the regulation of transcript and protein stability across species.

After neurogenesis, the first cell type of the mDA lineage found in the mouse is the mNbDA, a cell type with a transcriptional profile similar to that of hDA0 (Figure 1C,D). Indeed, both of these cell types express $N r 4 a 2$ and additional TFs that regulate the differentiation and survival of mDA neurons, such as $P b x 1$ (Villaescusa et al., 2016), Pitx3, and En1 (Veenvliet et al., 2013). In addition, $\mathrm{mNbDA}$ cells express the vesicular monoamine transporter (Slc18a2), also found in $\mathrm{mDA} 0, \mathrm{~m} / \mathrm{hDA} 1$, and $\mathrm{m} / \mathrm{hDA} 2$ (Figure 1D). However, $\mathrm{mNbDA}$ and hDA0 differ in that hDA0 cells express $T H$, whereas mNbDA cells do not.

The earliest post-mitotic cell types of the $\mathrm{mDA}$ lineage in mouse and human (mNbDA, mDA0, and hDA0), are relatively immature and do not express the dopamine transporter (Slc6a3) or Calbindin1 (Calb1), which in both species are found in the two mature embryonic mDA neurons, DA1 and DA2 (Figure 1D). Mouse and human DA1 neurons differ from the preceding DA0 neurons in their degree of maturation, as assessed by expression of Slc18a2 (Vmat2) and Slc6a3 (Dat). Additionally, DA2 neurons in both species express $A L D H 1 A$, an enzyme involved in the synthesis of retinoic acid (McCaffery and Drager, 1994), and LMO3, a TF highly enriched in the murine $\mathrm{SNc}$, which functions as a transcriptional co-regulator of PITX3 (Bifsha et al., 2017) and has also been suggested to play a role in Parkinson's disease (Briggs et al., 2015).

All mouse and human DA neurons (except hDA0) share the expression of $B N C 2$, a zinc finger TF primarily expressed in adult DA neurons of the SNc (Dougherty, 2017). This TF is known to regulate pigmentation of human keratinocytes (Jacobs et al., 2013) and GWAS studies have indicated that SNPs in this gene could be associated with Parkinson's disease (Hook et al., 2018). On the other hand, a consistent difference between mouse and human DA neurons (DA0-2) (Figure 1D) was the expression of significantly higher levels of doublecortin (DCX) in human neurons than in mouse. Since Dcx is a microtubule associated protein essential for neuronal migration (Gleeson et al., 1999), this result may reflect that human DA neurons need to migrate for longer distances than their mouse counterparts. Other genes that were differentially expressed in a species-specific manner in DA neurons were FOXA2, which was expressed in human but not murine DA1 neurons, and Lmo3, expressed in mouse but not human DA0. Overall, the DA neuron subtype that exhibited the greatest species differences was DA0, with mouse DA0 expressing TFs related to DA development/function at higher levels (Otx2, Wnt5a, Bnc2, and Lmo3) than human DA0 (Figures 1C,D). However, it should be noted that a set of late TFs that regulate $\mathrm{mDA}$ neuron differentiation (NR4A2, PBX1, PITX3, and EN1) were found in all DA neuron subtypes (DA0-2) (Figure 1D), suggesting the existence of very robust and common late differentiation mechanism in both species. 
In sum, while we found important species differences in the expression of early marker genes in proliferating cell types (Figure 1A), the expression of late marker genes in post-mitotic cell types varied less (Figure 1D). Furthermore, even within post-mitotic cells, gene expression in earlier homologous cell types, that is neuroblasts and DA0 neurons, varied more than in DA1-2, indicating that homologous differentiated cell types are more similar than homologous undifferentiated cell types. Finally, it is currently unclear which murine or human adult postnatal mDA neuron subtypes (Poulin et al., 2014, 2018; La Manno et al., 2016; Nichterwitz et al., 2016; Kee et al., 2017; Hook et al., 2018; Kramer et al., 2018; Zeisel et al., 2018; Tiklová et al., 2019a) are generated by each of the embryonic DA0-2 neurons and whether species differences may exist. Nevertheless, two lines of evidence suggest that embryonic DA2 neurons may be the precursors of SNc neurons in both species. First, the observation that DA2 neurons express several factors that are later found in the SNc, including Sox6 (Panman et al., 2014), ALDH1A1 (Galter et al., 2003), Rgs6 (Bifsha et al., 2014), and Lmo3 (Bifsha et al., 2017); and second, these genes have been associated to PD either by GWAS studies (SOX6, Dube et al., 2019), microarray data in PD post-mortem samples (LMO3, Briggs et al., 2015) or the presence of Parkinsonian symptoms in $\mathrm{Rgs}^{-/-}$mice (Luo et al., 2019). Ultimately, lineage tracing experiments using either human embryonic tissue or hES cells differentiated into midbrain cell types will be necessary to define the lineage relationship between embryonic and postnatal/adult human mDA neuron subtypes.

Taken together, analysis of the developing mouse and human $\mathrm{VM}$ at the single-cell level indicates that while a common pool of TFs is necessary to generate mDA neurons in both species, their expression is not always found in homologous cell types. This may lead to the formation of species-specific signaling complexes and transcriptional networks and/or their activation for different lengths of time, which may result in distinct signaling strengths and biological outputs in different species. These are intriguing possibilities that require further investigation.

\section{ANALYSIS OF DOPAMINERGIC DIFFERENTIATION OF HUMAN PLURIPOTENT STEM CELLS}

Advancements in the development of human stem cell-based cell replacement therapies for Parkinson's disease have recently led to hPSC-derived products entering the clinic, with clinical trials either in preparation (Studer, 2017; Sonntag et al., 2018; Barker et al., 2019) or currently underway (Takahashi and Price-Evans, 2019). However, the composition and quality of such cell preparations have not been examined in detail at the single-cell level. Therefore, it is currently unknown how well hPSC-derived cells resemble the endogenous fetal VM cell types previously used for transplantation. We previously performed a comprehensive scRNA-seq analysis of developing human fetal VM tissue, dissected in the same way and from the same stages as used for transplantation in PD patients. This data has been used as a standard to assess, in an unbiased manner, the cellular and molecular composition of hPSC preparations differentiated into midbrain cell types (La Manno et al., 2016). We believe that such datasets combined with machine learning methodologies will routinely be used in the near future to evaluate both the composition and quality of in vitro derived dopaminergic cell preparations to be used for transplantation in $\mathrm{PD}$ patients. Furthermore, such methods can be used to develop and establish the standards of cell quality necessary to qualify cell preparations for transplantation. In this section, we exclusively focus on the cellular composition of hPSC-derived mDA preparations in vitro, from a single cell perspective. For additional information on cell replacement therapy, including cell transplantation strategies, functionality, and outcomes in PD models, the reader is directed to existing reviews (Allan et al., 2010; Arenas et al., 2015a; Barker et al., 2015, 2019; Lindvall, 2016; Björklund and Lindvall, 2017; Studer, 2017).

In our analysis of human stem cell differentiation into $\mathrm{mDA}$ neurons (La Manno et al., 2016), stem cell preparations were differentiated using the protocol developed by Kriks et al. (2011). Cells were analyzed at different stages of differentiation to establish a trajectory from the pluripotent stem cell state, through the intermediate progenitor and neuroblast states, until the generation of dopaminergic neurons. This strategy allowed us to assess whether cells differentiating in vitro recapitulate key stages and cell types found in human embryonic midbrain development. The fidelity of the cell preparations was first determined by directly comparing the transcriptomes of the cells in the preparation to that of the cell types found in the developing human midbrain. In addition, supervised machine learning was used to identify key features of the endogenous human VM cell prototypes and to evaluate the stem cell products based on the probability of an individual cell being any of the human VM cell types (La Manno et al., 2016).

Direct comparison of the transcriptomes of cells differentiating in vitro to that of cells found in the developing midbrain in vivo revealed that hPSC-derived cells appeared to share a varying range of transcriptional similarity with the in vivo standards, indicating that the cellular identity of hPSC-derived cells is not as well resolved (La Manno et al., 2016). However, the magnitude of these differences was similar to that observed between endogenous human and mouse midbrain cell types during development, indicating that the overall similarity or quality of hPSC-derived cells obtained by the Kriks protocol is good, but that there might be some differences compared to the endogenous standards.

\section{Quality Differences Between Endogenous and hPSC-Derived Cell Types}

A detailed comparison between hPSC-derived and endogenous VM cell types by machine learning (using logistic regression) revealed significant differences between these two cell preparations. While human endogenous cells were all recognized as very similar to their respective prototypes as defined by machine learning (Figure 3A) and random gene expression was readily detected (Figure 3B), hPSC-derived cell types were 

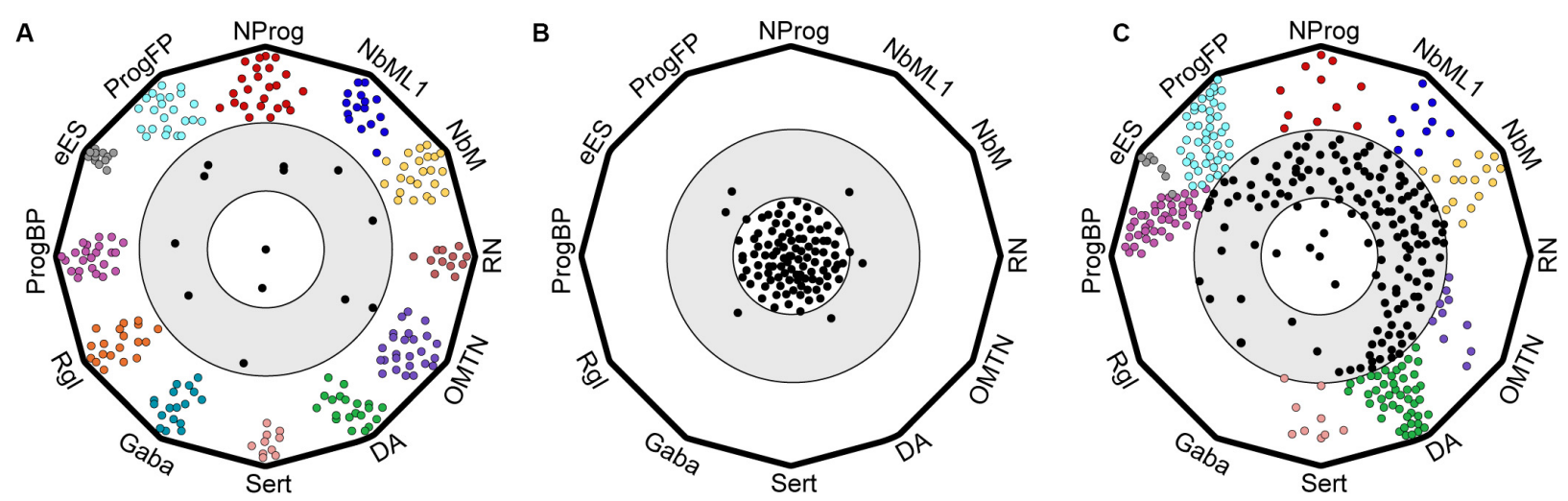

FIGURE 3 | Cartoon of polygon plots showing differences in cell type composition and quality between endogenous ventral midbrain and hPSC-derived midbrain cell types. Cells were evaluated by machine learning (logistic regression) as described in La Manno et al. (2016). (A) Endogenous ventral midbrain (VM) cell types used in the training dataset show similarity to their prototypes. Each vertex of the polygon defines a prototype and cells are represented as circles. Most endogenous VM cell types are in the outer circle, close to their prototypes. (B) Negative control showing randomized gene expression in cell types. Random cells cluster far away from the standards, in an inner circle, that defines cells with no midbrain identity. (C) hESCs differentiated into midbrain cell types with a protocol based on Kriks et al. (2011) give rise to well-defined midbrain cell types that resemble their in vivo prototypes (cells in outer circle), but also to less defined midbrain-like cells (intermediate gray zone) that are clearly distinct from non-midbrain cells (inner circle).

generally less well differentiated and exhibited more phenotypic variation (Figure $3 \mathrm{C}$ ). However, some of the cell types derived from hPSCs resembled their in vivo counterparts more closely. For instance, hPSCs generated high-quality human floor plate progenitors (hProgFP, a standard combining hProgFPL and hProgFPM) and good quality basal plate progenitors (hProgBP). At the neuroblast stage, fewer cells acquire a well-characterized $\mathrm{NbM}$ and NbML1 identity, but a good number of high quality dopaminergic neurons ( $\mathrm{hDA}$, a standard combining endogenous hDA0-2) are generated (Figure 3C). hPSC-derived $\mathrm{mDA}$ neurons expressed multiple key mDA markers such as NR4A2, $T H$, and $P B X 1$, but failed to express genes which commonly define hDA1-2, such as DAT/SLC6A3 and BNC2, or that define a specific embryonic mDA subtype, such as hDA2 (ALDH1A1). These differences may reflect the fact that the protocol used for the differentiation of hPSCs into mDA neurons (Kriks et al., 2011) does not mimic all aspects of in vivo mDA neuron development.

\section{Differences in Cell Transitions Between Endogenous and hPSC-Derived Cell Types}

Another feature that distinguished endogenous VM cell types from hPSC-derived cells in the machine learning analysis was the apparent difference in how differentiating cells transition from one intermediate cell type to another (La Manno et al., 2016). While each endogenous VM cell type was found to be very similar to its machine learning standard and cell types were clearly different from one another (Figure 3A), hPSC-derived cell types were less distinct and less well-defined along the differentiation axis; thus occupying an undefined gray zone in transcriptomic space (Figure 3C), which is distinct from cells in which the expression of midbrain genes was scrambled (Figure 3B) (La Manno et al., 2016). Since cells normally transition from one cell type to the next during embryonic development, e.g., from progenitor to neuroblast and finally to neuron, the clear separation between each endogenous cell type suggests that these transitions happen relatively fast in vivo and the probability of capturing them is rather low. Conversely, the presence of a continuum of cell types in hPSC-derived cultures suggests that such transitions may be much slower in vitro, as many cells appear to be captured while transitioning from one cell type to the next. Thus, it appears that endogenous midbrain progenitors differentiate following a sequence of events connecting well-defined and stable intermediate states through rapid transitions to generate the mature state. In contrast, cells differentiating from hPSCs in vitro go through a more constant differentiation flow characterized by less defined and less stable intermediate states. These differences in differentiation kinetics might be related to the lack of sufficient spatial information and the asynchronous nature of cells differentiated as a monolayer in two-dimensional (2D) cultures. This is in contrast with normal embryonic development, in which progenitor cells are immersed in a three-dimensional (3D) structure and surrounded by specific micro-environments, referred to as niches. These niches are composed of neighboring cells, which provide spatially arrayed information, such as mechanical forces and secreted factors, including morphogens, growth factors, extracellular matrix, neurotransmitters, and metabolites. It remains to be determined whether moving away from the traditional 2D culturing systems and toward 3D culturing systems may improve the molecular definition of individual cell types. Indeed, cells grown in 3D cultures tend to self-organize into discrete domains, and integrate more signals from neighboring cells (Kadoshima et al., 2013). In recent years, 3D culture systems have gained popularity and have been used to generate midbrain-like organoids containing functional dopaminergic neurons (Jo et al., 2016; Smits et al., 2019). Future experiments should thus aim to determine whether developmental transitions and overall cell 
quality can be improved by incorporating additional factors into the differentiation protocols and/or differentiating hPSCs as midbrain organoids.

\section{Differences in Cell Composition Between Endogenous and hPSC-Derived Cell Types: Therapeutic Implications}

In recent years, improved protocols for the derivation of functional mDA neurons from stem cell preparations have led to renewed interest in cell transplantation as a viable treatment option for Parkinson's disease. Nowadays, mDA neurons can be derived from hPSCs in a GMP-compliant manner (Doi et al., 2014; Kirkeby et al., 2017; Nolbrant et al., 2017). These efforts have recently culminated in the start of the first clinical trial involving hPSCs as a cell source for transplantation (Takahashi and Price-Evans, 2019). With the field moving quickly toward clinical applications, the ability to confidently assess the composition and quality of hPSCderived mDA preparations has become increasingly important. However, few studies have examined the composition of hPSCderived midbrain preparations at the single-cell level. In our study (La Manno et al., 2016), we used machine-learning analysis to assess our hPSC cultures differentiated toward an mDA fate using the Kriks protocol and found that these cells give rise to non-dopaminergic neurons, including serotonergic neurons and a few oculomotor and trochlear neurons (OMTN). However, other neuronal cell types such as red nucleus or GABAergic neurons were not identified (Figure 3C). More recently, Tiklová et al. (2019b) used scRNA-seq to analyze the general composition of hPSC derived midbrain cultures differentiated with a more recent protocol (Nolbrant et al., 2017) before and after transplantation in a rodent model of PD. Surprisingly, a large number of cells were identified as fibroblast-like vascular leptomeningeal cells (VLMCs), a cell type neither found in the developing human VM (La Manno et al., 2016), nor after transplantation of human fetal VM tissue in a rodent model of PD (Tiklová et al., 2019b). The functional impact of VLMCs on the rest of the transplanted cells and/or the host rodent brain remains to be explored. Similarly, the consequences of transplanting these cell types in a clinical setting are unknown since previous clinical trials were performed using human VM tissue, which does not contain VLMCs. Previous studies have suggested that the presence of non-midbrain cell types, such as serotonergic neurons, may underly the cause of graft-induced dyskinesias (Politis et al., 2010), arguing for the need to restrict cell transplantation to at least only those cell types present in the VM. It will therefore be of the utmost importance to determine the functional consequences of transplanting hPSC-derived preparations with and without VLMCs in animal models of PD prior to their application in cell replacement therapy for PD. We believe that a detailed analysis of the cellular and molecular composition of hPSC-derived preparations is necessary to progress toward a safe and efficient clinical application of stem cell-based cell replacement therapies for PD. In this context, we envision that scRNA-seq analysis will be increasingly used and required to assess the molecular composition of stem cell preparations and to determine whether the quality of cells in the preparation is comparable to that of the desired endogenous prototypes.

\section{How Can We Improve Current hPSCs Preparations for PD Cell Replacement Therapy?}

Concerning cell composition and cell quality, there are a few remaining challenges and some opportunities for improvement. To date, the challenge remains to achieve cell preparations containing only midbrain cell types or better yet, only the midbrain cell types required for cell replacement. Regarding the tissue, it is clear that cell preparations should exclusively contain cells present within the VM, the tissue that has demonstrated therapeutic value and provided proof of principle for cell replacement therapy in PD. However, future cell replacement strategies would be expected to achieve cell preparations further enriched in the cell type/s that need to be replaced and thus hold the greatest therapeutic value, such as precursors of $\mathrm{SNc}$ neurons. At the same time, it would be desirable to reduce the midbrain cell types that do not need to be replaced and do not contribute to the development, maintenance, or integration of the transplanted cells. With increasing knowledge of the cell composition and function of individual cell types during development and in the adult brain, we foresee the next step as being the generation of designer cell preparations containing the cell types required and sufficient to achieve the desired therapeutic effect without adverse effects. In this scenario, scRNA-seq and other single-cell technologies (discussed below) will have an increasingly important role. For instance, scRNA-seq analysis can be used in an iterative process where the information gained from the analysis and comparison of endogenous cell types with hPSC-derived cell types is used to guide the improvement of differentiation protocols. For example, the presence of non-midbrain cell types in the culture, or differences in gene expression identified in midbrain cell types derived from hPSCs compared to their in vivo counterparts can highlight genes and pathways that are aberrantly expressed and/or regulated in vitro, resulting in a change in cell composition or quality. Once these differences are identified, such genes or pathways could be targeted for activation or inhibition, so that the differentiation of stem cells is optimized to resemble endogenous brain standards as closely as possible.

The next challenges for cell replacement therapy in PD with regard to cell composition and quality will then be twofold. Firstly, subtype specification and late-stage maturation could be improved to generate cell preparations that are enriched in progenitor cells capable of selectively giving rise to $\mathrm{mDA}$ neurons of the ventral tier of the SNc. Secondly, those midbrain cell types unnecessary for transplantation should be identified and then eliminated from the culture so that only those cell types required for cell replacement would be transplanted. Additionally, future cell preparations could also be endowed with additional properties that may enhance their 
therapeutic value. For instance, by generating universal donor cells which cannot be targeted by the host immune system (Deuse et al., 2019), the need to immunosuppress the host would be eliminated. Another example would be the generation of cells resistant to the transmission of disease from the host brain to the transplanted cells, as has previously been described (Brundin et al., 2008); or the generation of cells resistant to disease by enhancing neuroprotective mechanisms and eliminating factors contributing to pathology. For such enhanced cells, we similarly believe that by systematically addressing specific pathways and molecular mechanisms at the single-cell level and by identifying which parameters are important for graft outcome, it may be possible to develop cell replacement therapy beyond the current state of the art and into the future.

\section{FUTURE OPPORTUNITIES}

Recent advances in the field of single-cell biology are making it possible to gain more information of not only gene expression but also gene regulation and function at the single cell level. For instance, it is now possible to sequence a much greater number of cells per sample at increased depth and resolution, thus increasing the information gained from each precious human fetal sample (Svensson et al., 2018). At the same time, new computational tools such as RNA velocity and scVelo allow to infer lineage relationships from scRNA-seq data (La Manno et al., 2018; Bergen et al., 2019). At the same time, genetic lineage tracing has evolved from using exogenous barcodes (Kester and van Oudenaarden, 2018) to the use of endogenous somatic mutations in mtDNA combined with scRNA-seq or ATAC-seq (Ludwig et al., 2019; $\mathrm{Xu}$ et al., 2019). During the last years, multiple spatial transcriptomics methods have also been developed, ranging from multiplexed single-molecule fluorescent in situ hybridization (FISH) methods, such as osmFISH (Codeluppi et al., 2018), seqFISH (Eng et al., 2019), and MERFISH (Xia et al., 2019); to sequencing-based methods, such as in situ sequencing (ISS) (Ke et al., 2013; Lee et al., 2014), Slide-seq (Rodriques et al., 2019), STARmap (Wang et al., 2018), and high definition spatial transcriptomics (HDST) (Vickovic et al., 2019), which provide positional information of molecularly defined cell types in a tissue.

In addition, several other single-cell methods that provide information about the cellular state beyond the transcriptome have recently been developed. In the field of single-cell epigenomics, several methods have now been developed that can be used to shed light on the epigenetic landscape, thus making it possible to gain information about the gene regulatory networks that govern lineage commitment and cell fate decisions during development. Several methods have been developed that provide information about chromatin accessibility, e.g., single-cell ATAC sequencing (Buenrostro et al., 2015) and single-cell hypersensitive site sequencing (scTHS-seq) (Lake et al., 2018), whereas methods such as single-cell chromatin immunoprecipitation sequencing (scChIP-seq)
(Rotem et al., 2015) and CUT\&Tag (Kaya-Okur et al., 2019) can elucidate chromatin state through histone modifications. Moreover, methods such as single-cell combinatorial indexed Hi-C (Sci-Hi-C) (Ramani et al., 2020) and DamID (Kind et al., 2015) can provide information about chromosome configuration and genome organization. In the near future, other methods in the emerging fields of single-cell proteomics and metabolomics are expected to shed additional light on cell phenotype at the single-cell level (Ali et al., 2019; Yang et al., 2019), thus allowing for a more dynamic picture of cellular heterogeneity. Finally, any information gained from such single-cell analysis will need to be validated through functional genomic analysis. CRISPR based genetic screens can be used to validate gene targets identified through single-cell analysis, either at the individual gene level or through pooled CRISPR screenings. Recently, several methods that couple pooled CRISPR screens with a single-cell transcriptomic read-out have been developed (Dixit et al., 2016; Jaitin et al., 2016; Datlinger et al., 2017; Feldman et al., 2019), making it possible to elucidate complex gene regulatory networks in a heterogeneous cell population such as the developing VM.

\section{CONCLUSION}

Until recently, most of our knowledge about $\mathrm{mDA}$ neuron development has come from studies in mice and little was known about the developmental programs that govern human mDA neuron development. Our scRNA-seq analysis of the developing human and murine VM has now generated a dataset that encompasses early mDA neuron development in both species, allowing us to study human development in an unbiased manner and to compare it to our current knowledge from the mouse (La Manno et al., 2016). From this analysis, we could identify several differences between human and murine VM development with regards to cell type composition, gene expression, and the temporal dynamics of VM development. Our results therefore highlight the need to use human midbrain development as the standard to tailor hPSCs-derived cells to the desired human prototypes, thus further improving the composition of cell preparations and the quality of the cells aimed for cell replacement in PD. It should also be noted that the single cell field is moving very rapidly into new technologies and it remains to be determined whether future scRNA-seq analysis of a greater number of cells from more samples at additional developmental time points and with greater sequencing depth may lead to the identification of additional cell types or subtypes, or to a better molecular definition of the cell types described in this review.

Future studies should aim to correlate the molecular signature of cell types identified by scRNA-seq in the mouse and human to their morphological properties (e.g., shape, size, dendrites and axonal projections), as well as their functional properties both in vitro and in animal models of PD (e.g., synaptic afferents and efferents, electrophysiological properties, neuronal activity and behavioral output). Moreover, by adopting a multi-omics approach and integrating different levels of information at the 
single-cell level, followed by functional genomics strategies for validation, it may be possible to understand and address the differences observed between the murine and human VM during development. Additionally, these same strategies could be used to identify differences between the healthy and Parkinsonian adult brain. Furthermore, these technologies will help us to identify and address any discrepancies between brain endogenous and stem cell-derived cell types, leading to the development of improved cell preparations and cell replacement therapy for the future treatment of PD.

\section{AUTHOR CONTRIBUTIONS}

All authors listed have made a substantial, direct and intellectual contribution to the work, and approved it for publication.

\section{REFERENCES}

Ali, A., Abouleila, Y., Shimizu, Y., Hiyama, E., Emara, S., Mashaghi, A., et al. (2019). Single-cell metabolomics by mass spectrometry: advances, challenges, and future applications. TrAC Trends Anal. Chem. 120:115436. doi: 10.1016/j. trac.2019.02.033

Allan, L. E., Petit, G. H., and Brundin, P. (2010). Cell transplantation in Parkinson's disease: problems and perspectives. Curr. Opin. Neurol. 23, 426-432. doi: 10. 1097/WCO.0b013e32833b1f62

Allen Brain Atlas. (2004). Allen Brain Atlas, Experiment79677369, Mouse P56, Cartpt-RP_071204_02_B05 -Specimen 335-1155, Sagittal, Level 18. Available online at: http://mouse.brain-map.org/experiment/show/79677369 (accessed May 17, 2020)

Almqvist, P. M., Akesson, E., Wahlberg, L. U., Pschera, H., Seiger, A., Sundstrom, E., et al. (1996). First trimester development of the human nigrostriatal dopamine system. Exp. Neurol. 139, 227-237. doi: 10.1006/exnr.1996.0096

Andersson, E., Jensen, J. B., Parmar, M., Guillemot, F., and Björklund, A. (2006a). Development of the mesencephalic dopaminergic neuron system is compromised in the absence of neurogenin 2. Development 133, 507-516. doi: 10.1242/dev.02224

Andersson, E., Tryggvason, U., Deng, Q., Friling, S., Alekseenko, Z., Robert, B., et al. (2006b). Identification of intrinsic determinants of midbrain dopamine neurons. Cell 124, 393-405. doi: 10.1016/j.cell.2005.10.037

Andersson, E. R., Saltó, C., Villaescusa, J. C., Cajanek, L., Yang, S., Bryjova, L., et al. (2013). Wnt5a cooperates with canonical Wnts to generate midbrain dopaminergic neurons in vivo and in stem cells. Proc. Natl. Acad. Sci. U.S.A. 110, E602-E610. doi: 10.1073/pnas.1208524110

Anthony, T. E., Mason, H. A., Gridley, T., Fishell, G., and Heintz, N. (2005). Brain lipid-binding protein is a direct target of Notch signaling in radial glial cells. Genes Dev. 19, 1028-1033. doi: 10.1101/gad.1302105

Arenas, E. (2010). Towards stem cell replacement therapies for Parkinson's disease. Biochem. Biophys. Res. Commun. 396, 152-156. doi: 10.1016/j.bbrc.2010.04.037

Arenas, E., Denham, M., and Villaescusa, J. C. (2015a). How to make a midbrain dopaminergic neuron. Development 142, 1918-1936. doi: 10.1242/dev.097394

Arenas, E., Saltó, C., and Villaescusa, C. (2015b). WNT signaling in midbrain dopaminergic neuron development and cell replacement therapies for Parkinson's disease. SpringerPlus 4, 1-32. doi: 10.1186/2193-1801-4-S1-L49

Artegiani, B., Jesus Domingues, A. M., Bragado Alonso, S., Brandl, E., Massalini, S., Dahl, A., et al. (2015). Tox: a multifunctional transcription factor and novel regulator of mammalian corticogenesis. EMBO J. 34, 896-910. doi: 10.15252/ embj.201490061

Ashique, A. M., Choe, Y., Karlen, M., May, S. R., Phamluong, K., Solloway, M. J., et al. (2009). The Rfx4 transcription factor modulates Shh signaling by regional control of ciliogenesis. Sci. Signal. 2:ra70. doi: 10.1126/scisignal.2000602

\section{FUNDING}

Financial support was obtained from Swedish Research Council (VR project 2016-01526), Swedish Foundation for Strategic Research (SB16-0065), European Commission (NeuroStemcellReconstruct, 874758), Knut and Alice Wallenberg Foundation (2018.0232), Karolinska Institutet (SFO: Strat. Regen. 2018 and Strat. Neuro. 2020), Chan Zuckerberg Initiative DAF and Silicon Valley Community Foundation (2018-191929), Hjärnfonden (FO2019-0068), and Cancerfonden (CAN 2016/572) to EA; and a fellowship from KID to ESÁ.

\section{ACKNOWLEDGMENTS}

We thank members of the Arenas lab for helpful discussion and suggestions.

Barker, R. A., Drouin-Ouellet, J., and Parmar, M. (2015). Cell-based therapies for Parkinson disease-past insights and future potential. Nat. Rev. Neurol. 11, 492-503. doi: 10.1038/nrneurol.2015.123

Barker, R. A., Farrell, K., Guzman, N. V., He, X., Lazic, S. E., Moore, S., et al. (2019). Designing stem-cell-based dopamine cell replacement trials for Parkinson's disease. Nat. Med. 25, 1045-1053. doi: 10.1038/s41591-019-0507-2

Barker, R. A., Parmar, M., Studer, L., and Takahashi, J. (2017). Human trials of stem cell-derived dopamine neurons for Parkinson's disease: dawn of a new Era. Cell Stem Cell 21, 569-573. doi: 10.1016/j.stem.2017.09.014

Bayer, S. A., Wills, K. V., Triarhou, L. C., and Ghetti, B. (1995). Time of neuron origin and gradients of neurogenesis in midbrain dopaminergic neurons in the mouse. Exp. Brain Res. 105, 191-199. doi: 10.1007/BF00240955

Bergen, V., Lange, M., Peidli, S., Wolf, F. A., and Theis, F. J. (2019). Generalizing RNA velocity to transient cell states through dynamical modeling. bioRxiv [Preprint]. doi: 10.1101/820936 bioRxiv: 820936 ,

Bifsha, P., Balsalobre, A., and Drouin, J. (2017). Specificity of Pitx3-dependent gene regulatory networks in subsets of midbrain dopamine neurons. Mol. Neurobiol. 54, 4921-4935. doi: 10.1007/s12035-016-0040-y

Bifsha, P., Yang, J., Fisher, R. A., and Drouin, J. (2014). Rgs6 is required for adult maintenance of dopaminergic neurons in the ventral substantia Nigra. PLoS Genet. 10:e1004863. doi: 10.1371/journal.pgen.1004863

Björklund, A., and Lindvall, O. (2017). Replacing dopamine neurons in Parkinson's disease: how did it happen? J. Parkinsons Dis. 7, S23-S33. doi: 10.3233/JPD179002

Blaess, S., and Ang, S. L. (2015). Genetic control of midbrain dopaminergic neuron development. Wiley Interdiscip. Rev. Dev. Biol. 4, 113-134. doi: 10.1002/ wdev.169

Blaess, S., Bodea, G. O., Kabanova, A., Chanet, S., Mugniery, E., Derouiche, A., et al. (2011). Temporal-spatial changes in Sonic Hedgehog expression and signaling reveal different potentials of ventral mesencephalic progenitors to populate distinct ventral midbrain nuclei. Neural Dev. 6:29. doi: 10.1186/17498104-6-29

Bodea, G. O., and Blaess, S. (2015). Establishing diversity in the dopaminergic system. FEBS Lett. 589, 3773-3785. doi: 10.1016/j.febslet.2015.09.016

Bonilla, S., Hall, A. C., Pinto, L., Attardo, A., Götz, M., Huttner, W. B., et al. (2008). Identification of midbrain floor plate radial glia-like cells as dopaminergic progenitors. Glia 56, 809-820. doi: 10.1002/glia.20654

Brichta, L., Greengard, P., and Flajolet, M. (2013). Advances in the pharmacological treatment of Parkinson's disease: targeting neurotransmitter systems. Trends Neurosci. 36, 543-554. doi: 10.1016/j.tins.2013.06.003

Briggs, C. E., Wang, Y., Kong, B., Woo, T. U. W., Iyer, L. K., and Sonntag, K. C. (2015). Midbrain dopamine neurons in Parkinson's disease exhibit a dysregulated miRNA and target-gene network. Brain Res. 1618, 111-121. doi: 10.1016/j.brainres.2015.05.021 
Brignani, S., and Pasterkamp, R. J. (2017). Neuronal subset-specific migration and axonal wiring mechanisms in the developing midbrain dopamine system. Front. Neuroanat. 11:55. doi: 10.3389/fnana.2017.00055

Brischoux, F., Griffond, B., Fellmann, D., and Risold, P. Y. (2002). Early and transient ontogenetic expression of the cocaine- and amphetamine-regulated transcript peptide in the rat mesencephalon: correlation with tyrosine hydroxylase expression. J. Neurobiol. 52, 221-229. doi: 10.1002/neu.10077

Broccoli, V., Boncinelli, E., and Wurst, W. (1999). The caudal limit of Otx2 expression positions the isthmic organizer. Nature 401, 164-168. doi: 10.1038/ 43670

Brodski, C., Blaess, S., Partanen, J., and Prakash, N. (2019). Crosstalk of intercellular signaling pathways in the generation of midbrain dopaminergic neurons in vivo and from stem cells. J. Dev. Biol. 7:3. doi: 10.3390/JDB7010003

Brown, A., Machan, J. T., Hayes, L., and Zervas, M. (2011). Molecular organization and timing of Wnt1 expression define cohorts of midbrain dopamine neuron progenitors in vivo. J. Comp. Neurol. 519, 2978-3000. doi: 10.1002/cne.22710

Brundin, P., Li, J. Y., Holton, J. L., Lindvall, O., and Revesz, T. (2008). Research in motion: the enigma of Parkinson's disease pathology spread. Nat. Rev. Neurosci. 9, 741-745. doi: 10.1038/nrn2477

Buenrostro, J. D., Wu, B., Litzenburger, U. M., Ruff, D., Gonzales, M. L., Snyder, M. P., et al. (2015). Single-cell chromatin accessibility reveals principles of regulatory variation. Nature 523, 486-490. doi: 10.1038/nature14590

Bye, C. R., Jönsson, M. E., Björklund, A., Parish, C. L., and Thompson, L. H. (2015). Transcriptome analysis reveals transmembrane targets on transplantable midbrain dopamine progenitors. Proc. Natl. Acad. Sci. U.S.A. 112, E1946-E1955. doi: 10.1073/pnas.1501989112

Castillo, S. O., Baffi, J. S., Palkovits, M., Goldstein, D. S., Kopin, I. J., Witta, J., et al. (1998). Dopamine biosynthesis is selectively abolished in substantia nigra/ventral tegmental area but not in hypothalamic neurons in mice with targeted disruption of the Nurr1 gene. Mol. Cell. Neurosci. 11, 36-46. doi: 10.1006/mcne.1998.0673

Chitalia, V. C., Foy, R. L., Bachschmid, M. M., Zeng, L., Panchenko, M. V., Zhou, M. I., et al. (2008). Jade-1 inhibits Wnt signalling by ubiquitylating $\beta$-catenin and mediates Wnt pathway inhibition by pVHL. Nat. Cell Biol. 10, 1208-1216. doi: $10.1038 /$ ncb1781

Codeluppi, S., Borm, L. E., Zeisel, A., La Manno, G., van Lunteren, J. A., Svensson, C. I., et al. (2018). Spatial organization of the somatosensory cortex revealed by osmFISH. Nat. Methods 15, 932-935. doi: 10.1038/s41592-018-0175-Z

Cohen, M., Briscoe, J., and Blassberg, R. (2013). Morphogen interpretation: the transcriptional logic of neural tube patterning. Curr. Opin. Genet. Dev. 23, 423-428. doi: 10.1016/j.gde.2013.04.003

Danielian, P. S., and McMahon, A. P. (1996). Engrailed-1 as a target of the Wnt-1 signalling pathway in vertebrate midbrain development. Nature 383, 332-334.

Datlinger, P., Rendeiro, A. F., Schmidl, C., Krausgruber, T., Traxler, P., Klughammer, J., et al. (2017). Pooled CRISPR screening with single-cell transcriptome readout. Nat. Methods 14, 297-301. doi: 10.1038/nmeth.4177

Dennis, D. J., Han, S., and Schuurmans, C. (2019). bHLH transcription factors in neural development, disease, and reprogramming. Brain Res. 1705, 48-65. doi: 10.1016/j.brainres.2018.03.013

Deuse, T., Hu, X., Gravina, A., Wang, D., Tediashvili, G., De, C., et al. (2019). Hypoimmunogenic derivatives of induced pluripotent stem cells evade immune rejection in fully immunocompetent allogeneic recipients. Nat. Biotechnol. 37 , 252-258. doi: 10.1038/s41587-019-0016-3

Dixit, A., Parnas, O., Li, B., Chen, J., Fulco, C. P., Jerby-Arnon, L., et al. (2016). Perturb-Seq: dissecting molecular circuits with scalable single-cell RNA profiling of pooled genetic screens. Cell 167, 1853-1866.e17. doi: 10.1016/j.cell. 2016.11.038

Doi, D., Samata, B., Katsukawa, M., Kikuchi, T., Morizane, A., Ono, Y., et al. (2014). Isolation of human induced pluripotent stem cell-derived dopaminergic progenitors by cell sorting for successful transplantation. Stem Cell Rep. 2, 337-350. doi: 10.1016/j.stemcr.2014.01.013

Dougherty, J. D. (2017). Generation and characterization of a mouse line for monitoring translation in dopaminergic neurons. Sci. Rep. 7:8117. doi: 10.1038/ s41598-017-08618-2

Dube, U., Ibanez, L., Budde, J. P., Benitez, B. A., Davis, A. A., Harari, O., et al. (2019). Overlapping genetic architecture between Parkinson disease and melanoma. Acta Neuropathol. 139, 347-364. doi: 10.1007/s00401-019-02110-z
Eng, C. H. L., Lawson, M., Zhu, Q., Dries, R., Koulena, N., Takei, Y., et al. (2019). Transcriptome-scale super-resolved imaging in tissues by RNA seqFISH+. Nature 568, 235-239. doi: 10.1038/s41586-019-1049-y

Fahn, S. (2003). Description of Parkinson's disease as a clinical syndrome. Ann. N. Y. Acad. Sci. 991, 1-14. doi: 10.1111/j.1749-6632.2003.tb07458.x

Feldman, D., Singh, A., Schmid-Burgk, J. L., Carlson, R. J., Mezger, A., Garrity, A. J., et al. (2019). Optical pooled screens in human cells. Cell 179, 787-799.e17. doi: 10.1016/j.cell.2019.09.016

Ferri, A. L. M., Lin, W., Mavromatakis, Y. E., Wang, J. C., Sasaki, H., Whitsett, J. A., et al. (2007). Foxa1 and Foxa2 regulate multiple phases of midbrain dopaminergic neuron development in a dosage-dependent manner. Development 134, 2761-2769. doi: 10.1242/dev.000141

Freeman, T. B., Spence, M. S., Boss, B. D., Spector, D. H., Strecker, R. E., Olanow, C. W., et al. (1991). Development of dopaminergic neurons in the human substantia nigra. Exp. Neurol. 113, 344-353. doi: 10.1016/0014-4886(91) 90025-8

Fu, H., Qi, Y., Tan, M., Cai, J., Takebayashi, H., Nakafuku, M., et al. (2002). Dual origin of spinal oligodendrocyte progenitors and evidence for the cooperative role of Olig2 and $\mathrm{Nkx} 2.2$ in the control of oligodendrocyte differentiation. Development 129, 681-693.

Galter, D., Buervenich, S., Carmine, A., Anvret, M., and Olson, L. (2003). ALDH1 mRNA: presence in human dopamine neurons and decreases in substantia nigra in Parkinson's disease and in the ventral tegmental area in schizophrenia. Neurobiol. Dis. 14, 637-647. doi: 10.1016/j.nbd.2003.09.001

German, D. C., Schlusselberg, D. S., and Woodward, D. J. (1983). Threedimensional computer reconstruction of midbrain dopaminergic neuronal populations: from mouse to man. J. Neural Transm. 57, 243-254. doi: 10.1007/ BF01248996

Gleeson, J. G., Peter, T. L., Flanagan, L. A., and Walsh, C. A. (1999). Doublecortin is a microtubule-associated protein and is expressed widely by migrating neurons. Neuron 23, 257-271. doi: 10.1016/S0896-6273(00)80778-3

Goldstein, D. S., Sullivan, P., Holmes, C., Miller, G. W., Alter, S., Strong, R., et al. (2013). Determinants of buildup of the toxic dopamine metabolite DOPAL in Parkinson's disease. J. Neurochem. 126, 591-603. doi: 10.1111/jnc.12345

Graham, V., Khudyakov, J., Ellis, P., and Pevny, L. (2003). SOX2 functions to maintain neural progenitor identity. Neuron 39, 749-765. doi: 10.1016/S08966273(03)00497-5

Hebsgaard, J. B., Nelander, J., Sabelström, H., Jönsson, M. E., Stott, S., and Parmar, M. (2009). Dopamine neuron precursors within the developing human mesencephalon show radial glial characteristics. Glia 57, 1648-1659. doi: 10. 1002/glia.20877

Hock, R., Furusawa, T., Ueda, T., and Bustin, M. (2007). HMG chromosomal proteins in development and disease. Trends Cell Biol. 17, 72-79. doi: 10.1016/ j.tcb.2006.12.001

Hook, P. W., McClymont, S. A., Cannon, G. H., Law, W. D., Morton, A. J., Goff, L. A., et al. (2018). Single-cell RNA-seq of mouse dopaminergic neurons informs candidate gene selection for sporadic Parkinson disease. Am. J. Hum. Genet. 102, 427-446. doi: 10.1016/j.ajhg.2018.02.001

Jacobs, L. C., Wollstein, A., Lao, O., Hofman, A., Klaver, C. C., Uitterlinden, A. G., et al. (2013). Comprehensive candidate gene study highlights UGT1A and BNC2 as new genes determining continuous skin color variation in Europeans. Hum. Genet. 132, 147-158. doi: 10.1007/s00439-012$1232-9$

Jaitin, D. A., Weiner, A., Yofe, I., Lara-Astiaso, D., Keren-Shaul, H., David, E., et al. (2016). Dissecting immune circuits by linking CRISPR-pooled screens with single-cell RNA-seq. Cell 167, 1883-1896.e15. doi: 10.1016/j.cell.2016.11.039

Jiang, Y. Q., and Oblinger, M. M. (1992). Differential regulation of bIII and other tubulin genes during peripheral and central neuron development. J. Cell Sci. $103,643-651$

Jo, J., Xiao, Y., Sun, A. X., Cukuroglu, E., Tran, H. D., Göke, J., et al. (2016). Midbrain-like organoids from human pluripotent stem cells contain functional dopaminergic and neuromelanin-producing neurons. Cell Stem Cell 19, 248-257. doi: 10.1016/j.stem.2016.07.005

Joksimovic, M., Anderegg, A., Roy, A., Campochiaro, L., Yun, B., Kittappa, R., et al. (2009). Spatiotemporally separable Shh domains in the midbrain define distinct dopaminergic progenitor pools. Proc. Natl. Acad. Sci. U.S.A. 106, 19185-19190. doi: $10.1073 /$ pnas. 0904285106 
Joyner, A. L., Liu, A., and Millet, S. (2000). Otx2, Gbx2 and Fgf8 interact to position and maintain a mid-hindbrain organizer. Curr. Opin. Cell Biol. 12, 736-741. doi: 10.1016/S0955-0674(00)00161-7

Kadkhodaei, B., Ito, T., Joodmardi, E., Mattsson, B., Rouillard, C., Carta, M., et al. (2009). Nurr1 is required for maintenance of maturing and adult midbrain dopamine neurons. J. Neurosci. 29, 15923-15932. doi: 10.1523/JNEUROSCI. 3910-09.2009

Kadoshima, T., Sakaguchi, H., Nakano, T., Soen, M., Ando, S., Eiraku, M., et al. (2013). Self-organization of axial polarity, inside-out layer pattern, and species-specific progenitor dynamics in human ES cell-derived neocortex. Proc. Natl. Acad. Sci. U.S.A. 110, 20284-20289. doi: 10.1073/pnas.131571 0110

Karus, M., Denecke, B., Ffrench-Constant, C., Wiese, S., and Faissner, A. (2011). The extracellular matrix molecule tenascin $\mathrm{C}$ modulates expression levels and territories of key patterning genes during spinal cord astrocyte specification. Development 138, 5321-5331. doi: 10.1242/dev.067413

Kawano, H., Ohyama, K., Kawamura, K., and Nagatsu, I. (1995). Migration of dopaminergic neurons in the embryonic mesencephalon of mice. Dev. Brain Res. 86, 101-113. doi: 10.1016/0165-3806(95)00018-9

Kaya-Okur, H. S., Wu, S. J., Codomo, C. A., Pledger, E. S., Bryson, T. D., Henikoff, J. G., et al. (2019). CUT\&Tag for efficient epigenomic profiling of small samples and single cells. Nat. Commun. 10:1930. doi: 10.1038/s41467-019-09982-5

Ke, R., Mignardi, M., Pacureanu, A., Svedlund, J., Botling, J., Wählby, C., et al. (2013). In situ sequencing for RNA analysis in preserved tissue and cells. Nat. Methods 10, 857-860. doi: 10.1038/nmeth.2563

Kee, N., Volakakis, N., Kirkeby, A., Dahl, L., Storvall, H., Nolbrant, S., et al. (2017). Single-cell analysis reveals a close relationship between differentiating dopamine and subthalamic nucleus neuronal lineages. Cell Stem Cell 20, 29-40. doi: 10.1016/j.stem.2016.10.003

Kefalopoulou, Z., Politis, M., Piccini, P., Mencacci, N., Bhatia, K., Jahanshahi, M., et al. (2014). Long-term clinical outcome of fetal cell transplantation for parkinson disease: two case reports. JAMA Neurol. 71, 83-87. doi: 10.1001/ jamaneurol.2013.4749

Kele, J., Simplicio, N., Ferri, A. L. M., Mira, H., Guillemot, F., Arenas, E., et al. (2006). Neurogenin 2 is required for the development of ventral midbrain dopaminergic neurons. Development 133, 495-505. doi: 10.1242/dev.02223

Kester, L., and van Oudenaarden, A. (2018). Single-cell transcriptomics meets lineage tracing. Cell Stem Cell 23, 166-179. doi: 10.1016/j.stem.2018.04.014

Kikuchi, T., Morizane, A., Doi, D., Magotani, H., Onoe, H., Hayashi, T., et al. (2017). Human iPS cell-derived dopaminergic neurons function in a primate Parkinson's disease model. Nature 548, 592-596. doi: 10.1038/nature23664

Kim, J. I., Ganesan, S., Luo, S. X., Wu, Y. W., Park, E., Huang, E. J., et al. (2015). Aldehyde dehydrogenase lal mediates a GABA synthesis pathway in midbrain dopaminergic neurons. Science 350, 102-106. doi: 10.1126/science.aac4690

Kind, J., Pagie, L., De Vries, S. S., Nahidiazar, L., Dey, S. S., Bienko, M., et al. (2015). Genome-wide maps of nuclear lamina interactions in single human cells. Cell 163, 134-147. doi: 10.1016/j.cell.2015.08.040

Kirkeby, A., Nolbrant, S., Tiklova, K., Heuer, A., Kee, N., Cardoso, T., et al. (2017). Predictive markers guide differentiation to improve graft outcome in clinical translation of hESC-based therapy for Parkinson's disease. Cell Stem Cell 20, 135-148. doi: 10.1016/j.stem.2016.09.004

Klar, A., Baldassare, M., and Jessell, T. M. (1992). F-spondin: a gene expressed at high levels in the floor plate encodes a secreted protein that promotes neural cell adhesion and neurite extension. Cell 69, 95-110. doi: 10.1016/0092-8674(92) 90121-R

Kornack, D. R., and Rakic, P. (1998). Changes in cell-cycle kinetics during the development and evolution of primate neocortex. Proc. Natl. Acad. Sci. U.S.A. 95, 1242-1246. doi: 10.1073/pnas.95.3.1242

Kramer, D. J., Risso, D., Kosillo, P., Ngai, J., and Bateup, H. S. (2018). Combinatorial expression of Grp and Neurod6 defines dopamine neuron populations with distinct projection patterns and disease vulnerability. Eneuro 5:ENEURO.0152-18.2018. doi: 10.1523/ENEURO.0152-18.2018

Kriks, S., Shim, J. W., Piao, J., Ganat, Y. M., Wakeman, D. R., Xie, Z., et al. (2011). Dopamine neurons derived from human ES cells efficiently engraft in animal models of Parkinson's disease. Nature 480, 547-551. doi: 10.1038/nature10648

La Manno, G., Gyllborg, D., Codeluppi, S., Nishimura, K., Salto, C., Zeisel, A., et al. (2016). Molecular diversity of midbrain development in mouse, human, and stem cells. Cell 167, 566-580.e19. doi: 10.1016/j.cell.2016.09.027
La Manno, G., Soldatov, R., Zeisel, A., Braun, E., Hochgerner, H., Petukhov, V., et al. (2018). RNA velocity of single cells. Nature 560, 494-498. doi: 10.1038/ s41586-018-0414-6

Lake, B. B., Chen, S., Sos, B. C., Fan, J., Kaeser, G. E., Yung, Y. C., et al. (2018). Integrative single-cell analysis of transcriptional and epigenetic states in the human adult brain. Nat. Biotechnol. 36, 70-80. doi: 10.1038/nbt. 4038

Lee, J. H., Daugharthy, E. R., Scheiman, J., Kalhor, R., Yang, J. L., Ferrante, T. C., et al. (2014). Highly multiplexed subcellular RNA sequencing in situ. Science 343, 1360-1363. doi: 10.1126/science. 1250212

Lees, A. J., Hardy, J., and Revesz, T. (2009). Parkinson's disease. Lancet 373, 2055-2066. doi: 10.1016/S0140-6736(09)60492-X

Lehnen, D., Barral, S., Cardoso, T., Grealish, S., Heuer, A., Smiyakin, A., et al. (2017). IAP-based cell sorting results in homogeneous transplantable dopaminergic precursor cells derived from human pluripotent stem cells. Stem Cell Rep. 9, 1207-1220. doi: 10.1016/j.stemcr.2017.08.016

Lehre, K. P., Levy, L. M., Ottersen, O. P., Storm-Mathisen, J., and Danbolt, N. C. (1995). Differential expression of two glial glutamate transporters in the rat brain: quantitative and immunocytochemical observations. J. Neurosci. 15, 1835-1853. doi: 10.1523/jneurosci.15-03-01835.1995

Lin, L., Rao, Y., and Isacson, O. (2005). Netrin-1 and slit-2 regulate and direct neurite growth of ventral midbrain dopaminergic neurons. Mol. Cell. Neurosci. 28, 547-555. doi: 10.1016/j.mcn.2004.11.009

Lindvall, O. (2016). Clinical translation of stem cell transplantation in Parkinson's disease. J. Int. Med. 279, 30-40. doi: 10.1111/joim.12415

Lindvall, O., and Björklund, A. (2011). Cell therapeutics in Parkinson's Disease. Neurotherapeutics 8, 539-548. doi: 10.1007/s13311-011-0069-6

Linnarsson, S., and Teichmann, S. A. (2016). Single-cell genomics: coming of age. Genome Biol. 17:97. doi: 10.1186/s13059-016-0960-x

Livesey, F. J., and Hunt, S. P. (1997). Netrin and netrin receptor expression in the embryonic mammalian nervous system suggests roles in retinal, striatal, nigral, and cerebellar development. Mol. Cell. Neurosci. 8, 417-429. doi: 10.1006/mcne. 1997.0598

Ludwig, L. S., Lareau, C. A., Ulirsch, J. C., Christian, E., Muus, C., Li, L. H., et al. (2019). Lineage tracing in humans enabled by mitochondrial mutations and single-cell genomics. Cell 176, 1325-1339.e22. doi: 10.1016/j.cell.2019.01.022

Luo, Z., Ahlers-Dannen, K. E., Spicer, M. M., Yang, J., Alberico, S., Stevens, H. E., et al. (2019). Age-dependent nigral dopaminergic neurodegeneration and $\alpha$-synuclein accumulation in RGS6-deficient mice. JCI Insight 4:e126769. doi: $10.1172 /$ jci.insight. 126769

Marklund, U., Alekseenko, Z., Andersson, E., Falci, S., Westgren, M., Perlmann, T., et al. (2014). Detailed expression analysis of regulatory genes in the early developing human neural tube. Stem Cells Dev. 23, 5-15. doi: 10.1089/scd.2013. 0309

Martínez, S. (2001). The isthmic organizer and brain regionalization. Int. J. Dev. Biol. 45, 367-371. doi: 10.1387/ijdb.11291867

Maxwell, S. L., Ho, H. Y., Kuehner, E., Zhao, S., and Li, M. (2005). Pitx3 regulates tyrosine hydroxylase expression in the substantia nigra and identifies a subgroup of mesencephalic dopaminergic progenitor neurons during mouse development. Dev. Biol. 282, 467-479. doi: 10.1016/j.ydbio.2005.03.028

McCaffery, P., and Drager, U. C. (1994). High levels of a retinoic acid-generating dehydrogenase in the meso- telencephalic dopamine system. Proc. Natl. Acad. Sci. U.S.A. 91, 7772-7776. doi: 10.1073/pnas.91.16.7772

McMahon, A. P., and Bradley, A. (1990). The Wnt-1 (int-1) proto-oncogene is required for development of a large region of the mouse brain. Cell 62, 1073-1085. doi: 10.1016/0092-8674(90)90385-R

Miller, G. W., Gainetdinov, R. R., Levey, A. I., and Caron, M. G. (1999). Dopamine transporters and neuronal injury. Trends Pharmacol. Sci. 20, 424-429. doi: 10.1016/S0165-6147(99)01379-6

Millet, S., Bloch-Gallego, E., Simeone, A., and Alvarado-Mallart, R. M. (1996). The caudal limit of Otx2 gene expression as a marker of the midbrain/hindbrain boundary: a study using in situ hybridisation and chick/quail homotopic grafts. Development 122, 3785-3797.

Millet, S., Campbell, K., Epstein, D. J., Losos, K., Harris, E., and Joyner, A. L. (1999). A role for $\mathrm{Gbx} 2$ in repression of Otx2 and positioning the mid/hindbrain organizer. Nature 401, 161-164. doi: 10.1038/43664

Molinoff, P. B., and Axelrod, J. (1971). Biochemistry of Catecholamines. Available online at: www.annualreviews.org (accessed February 2, 2020). 
Moreno-Bravo, J. A., Perez-Balaguer, A., Martinez, S., and Puelles, E. (2010). Dynamic expression patterns of Nkx6.1 and Nkx6.2 in the developing mesdiencephalic basal plate. Dev. Dyn. 239, 2094-2101. doi: 10.1002/dvdy.22327

Nakamura, H., Sato, T., and Suzuki-Hirano, A. (2008). Isthmus organizer for mesencephalon and metencephalon. Dev. Growth Differ. 50(Suppl. 1), S113-S118. doi: 10.1111/j.1440-169X.2008.00995.x

Nelander, J., Hebsgaard, J. B., and Parmar, M. (2009). Organization of the human embryonic ventral mesencephalon. Gene Expr. Patterns 9, 555-561. doi: 10. 1016/j.gep.2009.10.002

Nelson, E. L., Liang, C. L., Sinton, C. M., and German, D. C. (1996). Midbrain dopaminergic neurons in the mouse: computer-assisted mapping. J. Comp. Neurol. 369, 361-371. doi: 10.1002/(SICI)1096-9861(19960603)369:3<361: AID-CNE3<3.0.CO;2-3

Nichterwitz, S., Chen, G., Aguila Benitez, J., Yilmaz, M., Storvall, H., Cao, M., et al. (2016). Laser capture microscopy coupled with Smart-seq2 for precise spatial transcriptomic profiling. Nat. Commun. 7:12139. doi: 10.1038/ncomms 12139

Niclis, J. C., Gantner, C. W., Alsanie, W. F., McDougall, S. J., Bye, C. R., Elefanty, A. G., et al. (2017). Efficiently specified ventral midbrain dopamine neurons from human pluripotent stem cells under xeno-free conditions restore motor deficits in Parkinsonian rodents. Stem Cells Trans. Med. 6, 937-948. doi: 10 5966/sctm.2016-0073

Nolbrant, S., Heuer, A., Parmar, M., and Kirkeby, A. (2017). Generation of high-purity human ventral midbrain dopaminergic progenitors for in vitro maturation and intracerebral transplantation. Nat. Protoc. 12, 1962-1979. doi: 10.1038/nprot.2017.078

Omodei, D., Acampora, D., Mancuso, P., Prakash, N., Di Giovannantonio, L. G., Wurst, W., et al. (2008). Anterior-posterior graded response to Otx2 controls proliferation and differentiation of dopaminergic progenitors in the ventral mesencephalon. Development 135, 3459-3470. doi: 10.1242/dev. 027003

Ono, Y., Nakatani, T., Sakamoto, Y., Mizuhara, E., Minaki, Y., Kumai, M. et al. (2007). Differences in neurogenic potential in floor plate cells along an anteroposterior location: midbrain dopaminergic neurons originate from mesencephalic floor plate cells. Development 134, 3213-3225. doi: 10.1242/dev. 02879

Ostrem, B., Di Lullo, E., and Kriegstein, A. (2017). oRGs and mitotic somal translocation - a role in development and disease. Curr. Opin. Neurobiol. 42, 61-67. doi: 10.1016/j.conb.2016.11.007

Pakkenberg, B., Moller, A., Gundersen, H. J. G., Dam, A. M., and Pakkenberg, H. (1991). The absolute number of nerve cells in substantia nigra in normal subjects and in patients with Parkinson's disease estimated with an unbiased stereological method. J. Neurol. Neurosurg. Psychiatry 54, 30-33. doi: 10.1136/ jnnp.54.1.30

Panman, L., Papathanou, M., Laguna, A., Oosterveen, T., Volakakis, N., Acampora, D., et al. (2014). Sox6 and Otx2 control the specification of substantia nigra and ventral tegmental area dopamine neurons. Cell Rep. 8, 1018-1025. doi: 10.1016/j.celrep.2014.07.016

Placzek, M., and Briscoe, J. (2005). The floor plate: multiple cells, multiple signals. Nat. Rev. Neurosci. 6, 230-240. doi: 10.1038/nrn 1628

Poewe, W., Seppi, K., Tanner, C. M., Halliday, G. M., Brundin, P., Volkmann, J., et al. (2017). Parkinson disease. Nat. Rev. Dis. Primers 3:17013. doi: 10.1038/ nrdp. 2017.13

Politis, M., Wu, K., Loane, C., Quinn, N. P., Brooks, D. J., Rehncrona, S., et al. (2010). Serotonergic neurons mediate dyskinesia side effects in Parkinson's patients with neural transplants. Sci. Trans. Med. 2:38ra46. doi: 10.1126/ scitranslmed.3000976

Poulin, J. F., Caronia, G., Hofer, C., Cui, Q., Helm, B., Ramakrishnan, C., et al. (2018). Mapping projections of molecularly defined dopamine neuron subtypes using intersectional genetic approaches. Nat. Neurosci. 21, 1260-1271. doi: 10. 1038/s41593-018-0203-4

Poulin, J. F., Gaertner, Z., Moreno-Ramos, O. A., and Awatramani, R. (2020). Classification of midbrain dopamine neurons using single-cell gene expression profiling approaches. Trends Neurosci. 43, 155-169. doi: 10.1016/j.tins.2020. 01.004

Poulin, J. F., Zou, J., Drouin-Ouellet, J., Kim, K. Y. A., Cicchetti, F., and Awatramani, R. B. (2014). Defining midbrain dopaminergic neuron diversity by single-cell gene expression profiling. Cell Rep. 9, 930-943. doi: 10.1016/j.celrep. 2014.10.008

Poulsen, K. T., Armanini, M. P., Klein, R. D., Hynes, M. A., Phillips, H. S., and Rosenthal, A. (1994). TGF $\beta 2$ and TGF $\beta 3$ are potent survival factors for midbrain dopaminergic neurons. Neuron 13, 1245-1252. doi: 10.1016/08966273(94)90062-0

Prakash, N., Brodski, C., Naserke, T., Puelles, E., Gogoi, R., Hall, A., et al. (2006). A Wnt1-regulated genetic network controls the identity and fate of midbraindopaminergic progenitors in vivo. Development 133, 89-98. doi: 10.1242/dev. 02181

Prakash, N., Puelles, E., Freude, K., Trümbach, D., Omodei, D., Di Salvio, M., et al. (2009). Nkx6-1 controls the identity and fate of red nucleus and oculomotor neurons in the mouse midbrain. Development 136, 2545-2555. doi: 10.1242/ dev.031781

Puelles, E., Annino, A., Tuorto, F., Usiello, A., Acampora, D., Czerny, T., et al. (2004). Otx2 regulates the extent, identity and fate of neuronal progenitor domains in the ventral midbrain. Development 131, 2037-2048. doi: 10.1242/ dev.01107

Puelles, L., and Verney, C. (1998). Early neuromeric distribution of tyrosine-hydroxylase-immunoreactive neurons in human embryos. J. Comp. Neurol. 394, 283-308. doi: 10.1002/(SICI)1096-9861(19980511)394:3<283: AID-CNE2<3.0.CO;2-Y

Ramani, V., Deng, X., Qiu, R., Lee, C., Disteche, C. M., Noble, W. S., et al. (2020). Sci-Hi-C: a single-cell Hi-C method for mapping 3D genome organization in large number of single cells. Methods 170, 61-68. doi: 10.1016/j.ymeth.2019. 09.012

Rodriques, S. G., Stickels, R. R., Goeva, A., Martin, C. A., Murray, E., Vanderburg, C. R., et al. (2019). Slide-seq: a scalable technology for measuring genomewide expression at high spatial resolution. Science 363, 1463-1467. doi: 10.1126/ science.aaw1219

Rotem, A., Ram, O., Shoresh, N., Sperling, R. A., Goren, A., Weitz, D. A., et al. (2015). Single-cell ChIP-seq reveals cell subpopulations defined by chromatin state. Nat. Biotechnol. 33, 1165-1172. doi: 10.1038/nbt.3383

Samata, B., Doi, D., Nishimura, K., Kikuchi, T., Watanabe, A., Sakamoto, Y., et al. (2016). Purification of functional human ES and iPSC-derived midbrain dopaminergic progenitors using LRTM1. Nat. Commun. 7:13097. doi: 10.1038/ ncomms13097

Saucedo-Cardenas, O., Quintana-Hau, J. D., Le, W. D., Smidt, M. P., Cox, J. J., De Mayo, F., et al. (1998). Nurr1 is essential for the induction of the dopaminergic phenotype and the survival of ventral mesencephalic late dopaminergic precursor neurons. Proc. Natl. Acad. Sci. U.S.A. 95, 4013-4018. doi: 10.1073/pnas.95.7.4013

Saunders, A., Macosko, E. Z., Wysoker, A., Goldman, M., Krienen, F. M., de Rivera, H., et al. (2018). Molecular diversity and specializations among the cells of the adult mouse brain. Cell 174, 1015-1030.e16. doi: 10.1016/j.cell.2018.07.028

Schapira, A. H. V., Chaudhuri, K. R., and Jenner, P. (2017). Non-motor features of Parkinson disease. Nat. Rev. Neurosci. 18, 435-450. doi: 10.1038/nrn.2017.62

Sharma, K., Sheng, H. Z., Lettieri, K., Li, H., Karavanov, A., Potter, S., et al. (1998). LIM homeodomain factors Lhx3 and Lhx4 assign subtype identities for motor neurons. Cell 95, 817-828. doi: 10.1016/s0092-8674(00)81704-3

Smidt, M. P. (2017). Molecular programming of mesodiencephalic dopaminergic neuronal subsets. Front. Neuroanat. 11:59. doi: 10.3389/fnana.2017.00059

Smidt, M. P., Smits, S. M., Bouwmeester, H., Hamers, F. P. T., van der Linden, A. J. A., Hellemons, A. J. C. G. M., et al. (2004). Early developmental failure of substantia nigra dopamine neurons in mice lacking the homeodomain gene Pitx3. Development 131, 1145-1155. doi: 10.1242/dev.01022

Smits, L. M., Reinhardt, L., Reinhardt, P., Glatza, M., Monzel, A. S., Stanslowsky, N., et al. (2019). Modeling Parkinson's disease in midbrain-like organoids. Npj Parkinsons Dis. 5:5. doi: 10.1038/s41531-019-0078-4

Söllner, T., Bennett, M. K., Whiteheart, S. W., Scheller, R. H., and Rothman, J. E. (1993). A protein assembly-disassembly pathway in vitro that may correspond to sequential steps of synaptic vesicle docking, activation, and fusion. Cell 75, 409-418. doi: 10.1016/0092-8674(93)90376-2

Sonntag, K. C., Song, B., Lee, N., Jung, J. H., Cha, Y., Leblanc, P., et al. (2018). Pluripotent stem cell-based therapy for Parkinson's disease: current status and future prospects. Prog. Neurobiol. 168, 1-20. doi: 10.1016/j.pneurobio.2018. 04.005 
Studer, L. (2017). Strategies for bringing stem cell-derived dopamine neurons to the clinic-the NYSTEM trial. Prog. Brain Res. 230, 191-212. doi: 10.1016/bs. pbr.2017.02.008

Svensson, V., Vento-Tormo, R., and Teichmann, S. A. (2018). Exponential scaling of single-cell RNA-seq in the past decade. Nat. Protoc. 13, 599-604. doi: 10.1038/ nprot.2017.149

Takahashi, J., and Price-Evans, A. (2019). Preparing for first human trial of induced pluripotent stem cell-derived cells for Parkinson's disease: an interview with Jun Takahashi. Regen. Med. 14, 93-95. doi: 10.2217/rme-2018-0158

Thomas, K. R., and Capecchi, M. R. (1990). Targeted disruption of the murine int1 proto-oncogene resulting in severe abnormalities in midbrain and cerebellar development. Nature 346, 847-850. doi: 10.1038/346847a0

Tiklová, K., Björklund, Å. K., Lahti, L., Fiorenzano, A., Nolbrant, S., Gillberg, L., et al. (2019a). Single-cell RNA sequencing reveals midbrain dopamine neuron diversity emerging during mouse brain development. Nat. Commun. 10:581. doi: 10.1038/s41467-019-08453-1

Tiklová, K., Nolbrant, S., Fiorenzano, A., Björklund, Å. K., Sharma, Y., Heuer, A., et al. (2019b). Single cell gene expression analysis reveals human stem cellderived graft composition in a cell therapy model of Parkinson's disease. bioRxiv [Preprint]. doi: 10.1101/720870 bioRxiv: 720870,

Torp, R., Danbolt, N. C., Babaie, E., BjØrås, M., Seeberg, E., Storm-Mathisen, J., et al. (1994). Differential expression of two glial glutamate transporters in the rat brain: an in situ hybridization study. Eur. J. Neurosci. 6, 936-942. doi: 10.1111/j.1460-9568.1994.tb00587.x

Veenvliet, J. V., dos Santos, M. T. M. A., Kouwenhoven, W. M., Von Oerthel, L., Lim, J. L., van der Linden, A. J. A., et al. (2013). Specification of dopaminergic subsets involves interplay of En1 and Pitx3. Development 140, 3373-3384. doi: $10.1242 /$ dev. 094565

Vernay, B., Koch, M., Vaccarino, F., Briscoe, J., Simeone, A., Kageyama, R., et al. (2005). Otx2 regulates subtype specification and neurogenesis in the midbrain. J. Neurosci. 25, 4856-4867. doi: 10.1523/JNEUROSCI.5158-04. 2005

Verney, C., Zecevic, N., and Puelles, L. (2001). Structure of longitudinal brain zones that provide the origin for the substantia nigra and ventral tegmental area in human embryos, as revealed by cytoarchitecture and tyrosine hydroxylase, calretinin, calbindin, and GABA immunoreactions. J. Comp. Neurol. 429, 22-44. doi: 10.1002/1096-9861(20000101)429:1<22::AID-CNE3<3.0.CO;2-X

Vickovic, S., Eraslan, G., Salmén, F., Klughammer, J., Stenbeck, L., Schapiro, D., et al. (2019). High-definition spatial transcriptomics for in situ tissue profiling. Nat. Methods 16, 987-990. doi: 10.1038/s41592-019-0548-y

Villaescusa, J. C., Li, B., Toledo, E. M., Rivetti di Val Cervo, P., Yang, S., Stott, S. R., et al. (2016). A PBX1 transcriptional network controls dopaminergic neuron development and is impaired in Parkinson's disease. EMBO J. 35, 1963-1978. doi: $10.15252 /$ embj. 201593725

Walther, E. U., Dichgans, M., Maricich, S. M., Romito, R. R., Yang, F., Dziennis, S., et al. (1998). Genomic sequences of aldolase C (Zebrin II) direct lacZ expression exclusively in non-neuronal cells of transgenic mice. Proc. Natl. Acad. Sci. U.S.A. 95, 2615-2620. doi: 10.1073/pnas.95.5.2615

Wang, X., Allen, W. E., Wright, M. A., Sylwestrak, E. L., Samusik, N., Vesuna, S., et al. (2018). Three-dimensional intact-tissue sequencing of single-cell transcriptional states. Science 361:eaat5691. doi: 10.1126/science.aat 5691
Wassarman, K. M., Lewandoski, M., Campbell, K., Joyner, A. L., Rubenstein, J. L. R., Martinez, S., et al. (1997). Specification of the anterior hindbrain and establishment of a normal mid/hindbrain organizer is dependent on Gbx2 gene function. Development 124, 2923-2934.

Wilson, L., and Maden, M. (2005). The mechanisms of dorsoventral patterning in the vertebrate neural tube. Dev. Biol. 282, 1-13. doi: 10.1016/j.ydbio.2005. 02.027

Workman, A. D., Charvet, C. J., Clancy, B., Darlington, R. B., and Finlay, B. L. (2013). Modeling transformations of neurodevelopmental sequences across mammalian species. J. Neurosci. 33, 7368-7383. doi: 10.1523/JNEUROSCI. $5746-12.2013$

Wurst, W., Bally-Cuif, L., and Bally-Cuif, L. (2001). Neural plate patterning: upstream and downstream of the isthmic organizer. Nat. Rev. Neurosci. 2, 99-108. doi: 10.1038/35053516

Xia, C., Babcock, H. P., Moffitt, J. R., and Zhuang, X. (2019). Multiplexed detection of RNA using MERFISH and branched DNA amplification. Sci. Rep. 9, 1-13. doi: 10.1038/s41598-019-43943-8

Xu, J., Nuno, K., Litzenburger, U. M., Qi, Y., Corces, M. R., Majeti, R., et al. (2019). Single-cell lineage tracing by endogenous mutations enriched in transposase accessible mitochondrial DNA. ELife 8:e45105. doi: 10.7554/eLife. 45105

Yang, J., Brown, A., Ellisor, D., Paul, E., Hagan, N., and Zervas, M. (2013). Dynamic temporal requirement of Wnt1 in midbrain dopamine neuron development. Development 140, 1342-1352. doi: 10.1242/dev.080630

Yang, L., George, J., and Wang, J. (2019). Deep profiling of cellular heterogeneity by emerging single-cell proteomic technologies. Proteomics 1900226. doi: 10.1002/ pmic. 201900226

Yang, S., Toledo, E. M., Rosmaninho, P., Peng, C., Uhlén, P., Castro, D. S., et al. (2018). A Zeb2-miR-200c loop controls midbrain dopaminergic neuron neurogenesis and migration. Commun. Biol. 1:75. doi: 10.1038/s42003-0180080-0

Zeisel, A., Hochgerner, H., Ernfors, P., Marklund, U., Linnarsson, S., Lö, P., et al. (2018). Molecular architecture of the mouse nervous system resource molecular architecture of the mouse nervous system. Cell 174, 999-1014.e22. doi: 10.1016/ j.cell.2018.06.021

Zervas, M., Millet, S., Ahn, S., and Joyner, A. L. (2004). Cell behaviors and genetic lineages of the mesencephalon and rhombomere 1. Neuron 43, 345-357. doi: 10.1016/j.neuron.2004.07.010

Zetterström, R. H., Solomin, L., Jansson, L., Hoffer, B. J., Olson, L., and Perlmann, T. (1997). Dopamine neuron agenesis in Nurr1-deficient mice. Science 276, 248-250. doi: $10.1126 /$ science.276.5310.248

Conflict of Interest: The authors declare that the research was conducted in the absence of any commercial or financial relationships that could be construed as a potential conflict of interest.

Copyright (c) 2020 Ásgrímsdóttir and Arenas. This is an open-access article distributed under the terms of the Creative Commons Attribution License (CC BY). The use, distribution or reproduction in other forums is permitted, provided the original author(s) and the copyright owner(s) are credited and that the original publication in this journal is cited, in accordance with accepted academic practice. No use, distribution or reproduction is permitted which does not comply with these terms. 